\title{
IMPROVED BOUNDS FOR STEIN'S SQUARE FUNCTIONS
}

\author{
SANGHYUK LEE KEITH M. ROGERS ANDREAS SEEGER
}

\begin{abstract}
We prove a weighted norm inequality for the maximal Bochner-Riesz operator and the associated square-function. This yields new $L^{p}\left(\mathbb{R}^{d}\right)$ bounds on classes of radial Fourier multipliers for $p \geq 2+4 / d$ with $d \geq 2$, as well as space-time regularity results for the wave and Schrödinger equations.
\end{abstract}

\section{INTRODUCTION}

Consider the Bochner-Riesz means of order $\alpha$ defined for Schwartz functions $f \in \mathcal{S}\left(\mathbb{R}^{d}\right)$ by

$$
\mathcal{R}_{t}^{\alpha} f(x)=\frac{1}{(2 \pi)^{d}} \int_{|\xi| \leq t}\left(1-\frac{|\xi|^{2}}{t^{2}}\right)^{\alpha} \widehat{f}(\xi) e^{\imath\langle x, \xi\rangle} d \xi,
$$

where $\widehat{f}(\xi)=\int f(y) e^{-\imath\langle y, \xi\rangle} d y$. In connection with questions regarding almost everywhere summability, and in analogy to classical Littlewood-Paley functions for Poisson-integrals, Stein [36] introduced a square function defined by

$$
G^{\alpha} f(x)=\left(\int_{0}^{\infty}\left|\frac{\partial}{\partial t} \mathcal{R}_{t}^{\alpha} f(x)\right|^{2} t d t\right)^{1 / 2} .
$$

One is interested in the inequality $\left\|G^{\alpha} f\right\|_{p} \lesssim\|f\|_{p}$, where $A \lesssim B$ denotes $A \leq C B$ with an unspecified constant independent of $f$. As $t \partial_{t} \widehat{\mathcal{R}_{t}^{\alpha}} f(\xi)=2 \alpha|\xi|^{2} / t^{2}\left(1-|\xi|^{2} / t^{2}\right)_{+}^{\alpha-1} \widehat{f}(\xi)$, one can consider the $L^{p}$ problem as a question regarding the boundedness of a vector valued singular integral operator involving Riesz means of order $\alpha-1$. It is known that $L^{p}$ boundedness for $1<p \leq 2$ holds if and only if $\alpha>d(1 / p-1 / 2)+1 / 2$ (see [40], [21]), however the problem is more interesting in the range $p>2$ for which the condition $\alpha>$ $\max \{1 / 2, d(1 / 2-1 / p)\}$ is known to be necessary and conjectured to be also sufficient. For $d=1$ many proofs of the conjecture are known, for one of them see [41]. The conjecture in two dimensions was proven by Carbery [3], and partial results for $p>\frac{2(d+1)}{d-1}, d \geq 3$, are in [8], 32]. Here we improve on the range in dimensions $d \geq 3$.

Theorem 1.1. Let $d \geq 2$ and $p \in\left[\frac{2(d+2)}{d}, \infty\right)$. Then

$$
\left\|G^{\alpha} f\right\|_{p} \lesssim\|f\|_{p}, \quad \alpha>d\left(\frac{1}{2}-\frac{1}{p}\right) .
$$

As in the related work by the first author [23] our main tool will be Tao's bilinear estimate [42] for the adjoint of the Fourier restriction operator. The square function result implies the currently known sharp $L^{p}$ estimates for the maximal Bochner-Riesz operator

Date: Revised version for PLMS, April 24, 2011.

2000 Mathematics Subject Classification. 42B15, 35B65.

Key words and phrases. Square functions, Bochner-Riesz means, Wave and Schrödinger semigroup, mixed norm estimates.

Supported in part by NRF grant 2009-0072531 (Korea), MICINN grant MTM2010-16518 (Spain) and NSF grant 0652890 (USA). 
obtained by Carbery [3] in two dimension and by the first author [23] in higher dimensions; however, as pointed out in [23], somewhat weaker estimates are already enough to bound the maximal function. More precisely, for a compact $t$-interval $I \subset(0, \infty)$, the estimate in 23] could be formulated as a variational $L^{p}\left(V_{4}(I)\right)$ inequality for Riesz means of order $\lambda>\lambda_{\text {crit }}:=d(1 / 2-1 / p)-1 / 2$, or a slightly better regularity result involving the Sobolev space $L_{1 / 4+\varepsilon}^{4}(I)$.

The $L^{p}$-estimate for the square function is significant for various reasons. Firstly, it yields regularity results for wave and Schrödinger operators which will be discussed below. Secondly, for compact $I \subset(0, \infty)$ it implies $L^{p}\left(V_{2}(I)\right)$ or $L^{p}\left(L_{1 / 2+\varepsilon}^{2}(I)\right)$ results for BochnerRiesz means of order $\lambda>\lambda_{\text {crit }}$. Thirdly, the $L^{p}$-result for $G^{\alpha}$ implies boundedness results for maximal operators associated with more general classes of radial Fourier multipliers as in [5], [13, and finally, an inequality by Carbery, Gasper and Trebels [6] relating radial multipliers and $G^{\alpha}$ yields the following sharp $L^{p} \rightarrow L^{p}$ boundedness result of HörmanderMikhlin type.

Corollary 1.2. Let $d \geq 2$ and $p \in\left(1, \frac{2(d+2)}{d+4}\right] \cup\left[\frac{2(d+2)}{d}, \infty\right)$. Let $\varphi$ be a nontrivial $C^{\infty}$ function compactly supported in $(0, \infty)$. Then

$$
\sup _{f \in \mathcal{S}:\|f\|_{p} \leq 1}\left\|\mathcal{F}^{-1}[m(|\cdot|) \widehat{f}]\right\|_{p} \lesssim \sup _{t>0}\|\varphi m(t \cdot)\|_{L_{\alpha}^{2}(\mathbb{R})}, \quad \alpha>d\left|\frac{1}{p}-\frac{1}{2}\right| .
$$

Weighted norm inequalities. More information about $G^{\alpha}$ can be obtained by considering an $L^{2}$ weighted norm inequality which involves a "universal" maximal operator $\mathfrak{V}_{q}$ acting on the weights, and which is strong enough to imply the above $L^{p}$ estimates. The operator $\mathfrak{V}_{q}$ is a maximal operator which is bounded on $L^{r}$ for $q<r \leq \infty$, and the $L^{p}$ estimates for $G^{\alpha}$ in Theorem 1.1 can be deduced after an additional interpolation if we take $q<(p / 2)^{\prime}$. An informal discussion of the definition of the weight operator is given below.

Theorem 1.3. Let $d \geq 2$ and $q \in\left(1, \frac{d+2}{2}\right)$. Then there is an operator $\mathfrak{V}_{q}$ which is bounded on $L^{r}$ for $q<r \leq \infty$, such that

$$
\int_{\mathbb{R}^{d}}\left|G^{\alpha} f(x)\right|^{2} w(x) d x \lesssim \int|f(x)|^{2} \mathfrak{V}_{q} w(x) d x, \quad \alpha>\frac{d}{2 q} .
$$

Moreover,

$$
\int_{\mathbb{R}^{d}} \sup _{t>0}\left|\mathcal{R}_{t}^{\lambda} f(x)\right|^{2} w(x) d x \lesssim \int|f(x)|^{2} \mathfrak{V}_{q} w(x) d x, \quad \lambda>\frac{d-q}{2 q} .
$$

The weighted inequalities (11) and (2) are motivated by one of Stein's problems in [38. It was asked whether the operator defining the weight on the right hand side of (2) could be chosen to be a Nikodym maximal operator (see also Córdoba [9] for a related question). This seems currently unknown. For the range $q \in\left(1, \frac{d+1}{2}\right]$, Christ [8] proved the weighted inequality with the simple weight $\left(M|w|^{q}\right)^{1 / q}$, where $M$ denotes the Hardy-Littlewood maximal operator. In two dimensions, Carbery [4] proved a weighted inequality with an operator $W_{2}$ in place of $\mathfrak{V}_{2}$, such that $W_{2}$ is bounded on $L^{r}\left(\mathbb{R}^{2}\right)$ for $r \in(2,4]$. The extension of that result with the weight operator bounded for $r \in(2, \infty]$ was established by Carbery and the third author [7].

We now give an informal description of the weight operator $\mathfrak{V}_{q}$ and refer to 92 for the precise description of a closely related operator $\mathfrak{W}_{q}$ (with a more technical definition) which will be of weak type $(q, q)$ and can be used instead of $\mathfrak{V}_{q}$. The reader may then check that $\mathfrak{V}_{q}$ satisfies $\mathfrak{V}_{q} \gtrsim \mathfrak{W}_{q}$ and is still bounded on $L^{r}$ for $r>q$. In $\$ 2$ we shall also prove a 
refinement of Theorem 1.3 which will be significant for endpoint bounds such as Theorem 1.4 below.

The weight $\mathfrak{V}_{q} w$ involves the sum of two maximal functions associated with tubes of large eccentricity;

$$
\mathfrak{V}_{q} w=\left(M\left[\sup _{\mathfrak{e} \geq 1} \mathfrak{e}^{-2\left(\frac{d}{q}-1\right)}\left(M V_{\mathfrak{e}} w+[\log (2+\mathfrak{e})]^{2} \sup _{l \in \mathbb{Z}} M V_{\mathfrak{e}, l, q}^{\operatorname{main}}\left(P_{l} w\right)\right)\right]^{1+\varepsilon}\right)^{\frac{1}{1+\varepsilon}},
$$

with some $\varepsilon>0$. Here $P_{l} w$ is a standard dyadic frequency cutoff localizing $\widehat{w}$ to frequencies of size $\approx 2^{l}$. The first summand is similar to the standard Nikodym maximal function, but much better behaved due to the small damping factor. For $\mathfrak{e} \geq 1$, the function $V_{\mathfrak{e}} w$ is the usual maximal function associated with the tubes (or cylinders) which are centered at the origin with eccentricity (defined as the quotient length/width) equal to $\mathfrak{e}$. The second summand involves the maximal function

$$
V_{\mathfrak{e}, l, q}^{\operatorname{main}} g(x)=\sup _{\theta \in S^{d-1}}\left(M_{\theta, \mathfrak{e}}\left[\sup _{\Psi}|\Psi * g|^{q}\right](x)\right)^{1 / q},
$$

where the supremum in $\Psi$ ranges over suitable classes of $L^{1}$-normalized Schwartz functions associated with tubes of length $2^{-l} \mathfrak{e}$ and width $2^{-l}$, in the direction of $\theta$. The maximal operator $M_{\theta, \mathfrak{e}}$ is associated with the tubes in the direction $\theta$, with fixed eccentricity $\mathfrak{e}$. This definition is reminiscent of the "grand maximal function" in the theory of Hardy spaces ([17]) as it involves a supremum over convolutions with kernels in a suitably normalized and rescaled class of Schwartz functions, and a significant gain is achieved when these kernels are convolved with functions that have a suitable cancellation property (such as $P_{l} w$ ).

Concerning the boundedness properties of $\mathfrak{V}_{q}$, for the first summand we shall only need a standard and non-optimal bound $\left\|V_{\mathfrak{e}}\right\|_{L^{q} \rightarrow L^{q}}=O\left(\mathfrak{e}^{a}\right)$ with $a>(d-2) / q$, for $q \geq 2$, and the small factor $\mathfrak{e}^{2-2 d / q}$ helps to get the claimed bound. For the main term the range cannot be improved as the maximal function involves powers $|\Phi * w|^{q}$. Here the terms with small eccentricity $(\approx 1)$ contribute most, and in order to establish the proper bounds for terms with large eccentricity one uses cancellation, namely the annular support property of $\widehat{P_{l} w}$.

Wave and Schrödinger operators. One can apply $L^{p}$ bounds for variants of the BochnerRiesz square-function to obtain regularity results for spherical means and solutions of the wave and Schrödinger equations. This application is suggested by a theorem of Kaneko and Sunouchi [22] relating $G^{\alpha}$ to another square function which was introduced by Stein in his study of spherical maximal operators (cf. [37]). Define the spherical mean of order $\beta>0$ by

$$
\mathcal{A}_{t}^{\beta} f(x)=\frac{\Gamma\left(\frac{d+2 \beta}{2}\right)}{\pi^{d / 2} \Gamma(\beta)} \int_{|y| \leq t} \frac{1}{t^{d}}\left(1-\frac{|y|^{2}}{t^{2}}\right)^{\beta-1} f(x-y) d y ;
$$

for smaller values of $\beta$ the definition can be extended by analytic continuation. In [22] an application of Plancherel's theorem with respect to $t$ is used to show that $G^{\alpha}$ is pointwise equivalent with a square function generated by spherical means, namely, for $\alpha>0$,

$$
G^{\alpha} f(x) \approx\left(\int_{0}^{\infty}\left|\frac{\partial}{\partial t} \mathcal{A}_{t}^{\alpha-\frac{d-2}{2}} f(x)\right|^{2} t d t\right)^{1 / 2}
$$

for all Schwartz functions $f$. Here we shall not use this equivalence explicitly but prove a closely related sharp $L^{p}\left(L^{2}\right)$ regularity result for solutions of the wave and Schrödinger equations with initial data in $L^{p}$ Sobolev spaces. In order to formulate a unifying result, for 
$a \in(0, \infty)$, we let $U_{t}^{a} f$ denote the solution to the initial value problem $\imath \partial_{t} u+\left(-\Delta_{x}\right)^{a / 2} u=0$ with $u(\cdot, 0)=f$;

$$
U_{t}^{a} f=\exp \left(\imath t(-\Delta)^{a / 2}\right) f .
$$

The case $a=2$ corresponds to the Schrödinger equation and the case $a=1$ to a wave equation.

Theorem 1.4. Let $d \geq 2, p \in\left(\frac{2(d+2)}{d}, \infty\right), a \in(0, \infty)$, and let $I$ be a compact time interval. Then

$$
\left\|\left(\int_{I}\left|U_{t}^{a} f\right|^{2} d t\right)^{1 / 2}\right\|_{p} \lesssim\|f\|_{L_{s}^{p}}, \quad \frac{s}{a}=d\left(\frac{1}{2}-\frac{1}{p}\right)-\frac{1}{2} .
$$

In fact this holds for initial data in the Besov space $B_{s, p}^{p}$ (which contains $L_{s}^{p}$ for $p \geq 2$ ).

One can also consider the same regularity problem in the mixed norm space $L^{p}\left(L^{q}(I)\right)$ with $q \in(2, \infty]$. For this range the analogy between the wave and Schrödinger equation breaks down (and some endpoint versions of the deeper 'local smoothing' result for the wave equation are currently available only in four and higher dimensions, cf. [20]). However, for $a \in(0,1) \cup(1, \infty)$ and $d \geq 2$, one can deduce sharp $B_{s, p}^{p} \rightarrow L^{p}\left(L^{q}(I)\right)$ estimates with $s=a d(1 / 2-1 / p)-a / q$, in the range $p \in(2+4 / d, \infty)$. This follows from a combination of Theorem 1.4 and the result in Appendix A. Moreover, one can, for a limited range of $q$, obtain further estimates for $p>2+4 /(d+1)$ and $d \geq 1$, essentially by interpolation with results in [29]; in dimensions $d \geq 2$ this currently requires the restriction $a>1$. These $L^{p}\left(L^{q}\right)$-estimates are stated in $\$ 6$, and a further, more substantial improvement for $d=2$ will be considered in [25].

Remark. After the first version of this paper was submitted for publication, Bourgain and Guth posted a preprint [2] containing very substantial improvements on the $L^{p}$ boundedness for oscillatory integrals related to the Fourier restriction problem, with implications for Bochner-Riesz multipliers. It would be of great interest to investigate the impact of their methods on Stein's square-function, weighted norm inequalities, and other issues discussed in this paper.

This paper. In $₫ 2$ we formulate a more precise weighted inequality (Theorem 2.1), and give the definitions and boundedness properties of suitable weight operators. In 33 we prove some $L^{2} \rightarrow L^{p}$ estimates for radial convolution operators and prepare for the proof of the weighted inequalities. These are established in $₫ 4$, In $\$ 5$ we prove $L^{p}\left(L^{2}\right)$ estimates for wave and Schrödinger equations and in $₫ 6$ we discuss some $L^{p}\left(L^{q}\right)$ bounds for $q>2$. Appendix $\mathrm{A}$ contains auxiliary results on combining inequalities for frequency localized operators.

Some notational references. For two nonnegative quantities $A, B$ the notation $A \lesssim B$, or $B \gtrsim A$, is used for $A \leq C B$, with some unspecified positive constant $C$. We also use $A \approx B$ to indicate that $A \lesssim B$ and $B \lesssim A$. To avoid unwieldy formulas we will sometimes shorten the notation for products involving a complex conjugate and use, given two complex terms $\mathcal{E}_{1}$ and $\mathcal{E}_{2}$, the expression $\prod_{i=1,2}^{*}\left[\mathcal{E}_{i}\right]=\mathcal{E}_{1} \overline{\mathcal{E}_{2}}$. For convolution operators given by Fourier multipliers $a(\xi)$ we occasionally use the symbol notation $a(D) f:=\mathcal{F}^{-1}[a \widehat{f}]$, where $\mathcal{F}^{-1}$ denotes the inverse Fourier transform. By $P_{n}, \widetilde{P}_{n}$ we denote dyadic frequency cutoff operators which localize to frequencies of size $\approx 2^{n}$, so that $P_{n} \widetilde{P}_{n}=P_{n}$, see 22 for the precise definition. 


\section{A STRONGER WEIGHTED NORM INEQUALITY}

We formulate a weighted norm inequality for a square function generated by thin pieces of the Bochner-Riesz multiplier. To fix notation, let $\phi$ be a Schwartz function supported in $(1 / 2,2)$ with the property that

$$
\left|\phi^{(\nu)}(t)\right| \leq 1, \quad \nu=0, \ldots, d+2 .
$$

Let $0<\delta<1 / 2$ and define the convolution operator $S_{t}^{\delta} \equiv S_{t}^{\delta, \phi}$ by

$$
\widehat{S_{t}^{\delta}} f(\xi)=\phi\left(\delta^{-1}\left(1-\frac{|\xi|^{2}}{t^{2}}\right)\right) \widehat{f}(\xi) .
$$

Assuming (5) we shall usually drop the superscript $\phi$, as our estimates will be understood to be uniform in $\phi$.

Theorem 2.1. Let $d \geq 2$ and $q \in\left(1, \frac{d+2}{2}\right)$. For $0<\delta<1 / 2$, there are operators $\mathfrak{W}_{q, \delta}$ defined on $L^{q}+L^{\infty}$, so that the weighted norm inequality

$$
\int_{\mathbb{R}^{d}} \int_{0}^{\infty}\left|S_{t}^{\delta} f(x)\right|^{2} \frac{d t}{t} w(x) d x \lesssim \delta^{2-d / q} \int_{\mathbb{R}^{d}}|f(x)|^{2} \mathfrak{W}_{q, \delta} w(x) d x
$$

holds for all $w \in L^{q}+L^{\infty}$ and the operators $\mathfrak{W}_{q, \delta}$ satisfy the following properties:

(i) The maximal operator defined by

$$
\mathfrak{W}_{q} w=\sup _{0<\delta<1 / 2} \mathfrak{W}_{q, \delta} w
$$

is of weak type $(q, q)$ and bounded on $L^{r}$ for $q<r \leq \infty$.

(ii) If $q \in\left[2, \frac{d+2}{2}\right)$, then the operators $\mathfrak{W}_{q, \delta}$ are bounded on $L^{q}$, uniformly in $\delta$. Moreover if $q \in(1,2)$ then $\left\|\mathfrak{W}_{q, \delta}\right\|_{L^{q} \rightarrow L^{q}} \lesssim\left[\log \left(\frac{1}{\delta}\right)\right]^{\frac{1}{q}-\frac{1}{2}}$.

We shall also consider local versions of (17) with the $t$-integral extended over a dyadic interval and for which the $L^{q}$ bounds of the corresponding weight operators are independent of $\delta$ for all $q \in\left(1, \frac{d+2}{2}\right)$, see Theorem 4.1 below.

To deduce inequality (11) with $\mathfrak{W}_{q}$ in place of $\mathfrak{V}_{q}$ one splits the multiplier into a part near the origin and a part near the unit sphere. The part near the origin is dealt with by the standard weighted norm inequality for singular integrals in [12. One then decomposes the part near the unit sphere into smooth multipliers supported on thin annuli of width $\delta=2^{-j}$, applies Theorem 2.1, and sums a geometric series. The maximal inequality (2) follows from (11) by well-known arguments in ([39, §VII.5]) together with a weighted norm inequality for the Hardy-Littlewood maximal function ([16]). If we take $q=(p / 2)^{\prime}$ then by duality and an application of the Marcinkiewicz interpolation theorem one obtains Theorem 1.1 for $p>2+4 / d$. Interpolation with an $L^{2}$ inequality yields the result also for $p=2+4 / d$. Theorem 2.1 also implies a sharp $L^{p}$ result for the square-functions generated by $S_{t}^{\delta}$ which is stated in Corollary 4.2 below.

Definition of $\mathfrak{W}_{q, \delta}$. We assume throughout this section that $d \geq 2$. The definition of $\mathfrak{W}_{q, \delta} w$ in (16) involves a suitably damped Nikodym maximal function and another (more important) maximal function acting on functions with Fourier transform supported away from the origin.

Let $0<\delta_{\circ}<1$, let $\theta$ be a unit vector in $\mathbb{R}^{d}$ and let

$$
R_{\delta_{\circ}, t}^{\theta}=\left\{y \in \mathbb{R}^{d}:|\langle y, \theta\rangle| \leq t,|y-\langle y, \theta\rangle \theta| \leq t \delta_{\circ}\right\} .
$$


Then the Nikodym maximal function associated with tubes of eccentricity $\delta_{\circ}$ is defined by

$$
\mathfrak{M}_{\delta_{\circ}} g(x)=\sup _{\theta \in S^{d-1}} \sup _{t>0} \frac{1}{\left|R_{\delta_{\circ}, t}^{\theta}\right|} \int_{R_{\delta_{0}, t}^{\theta}}|g(x+y)| d y .
$$

Now we describe our second maximal operator. Let $\mathcal{N} \geq d+3$ be a large positive integer and let $\mathcal{S}(\mathcal{N})$ be the set of all Schwartz functions $\psi$ for which

$$
\|\psi\|_{\mathcal{N}}:=\max _{|\alpha| \leq \mathcal{N}} \sup _{x}(1+|x|)^{\mathcal{N}}\left|\partial_{x}^{\alpha} \psi(x)\right| \leq 1 .
$$

The number $\mathcal{N}$ will be fixed throughout the paper and constants in inequalities will depend on $\mathcal{N}$ (one may want to choose $\mathcal{N}=d+3$ ).

For $j \geq 0$ and $\theta \in S^{d-1}$ let $\ell_{\theta, j}$ be the unique linear transformation defined by

$$
\ell_{\theta, j}(\theta)=2^{j} \theta, \quad \ell_{\theta, j}(y)=y \text { if }\langle\theta, y\rangle=0 .
$$

Then $\operatorname{det} \ell_{\theta, j}=2^{j}$. Let $\mathcal{S}_{n}^{\theta, j}$ be the set of all $\Psi$ for which $2^{-n d_{2} j} \Psi\left(\ell_{\theta, j} 2^{-n} \cdot\right)$ belongs to $\mathcal{S}(\mathcal{N})$. Typical examples of functions in $\mathcal{S}_{n}^{\theta, j}$ are $L^{1}$ normalized bump functions essentially supported on a tube with direction $\theta$, length $2^{j-n}$ and width $2^{-n}$. We define a maximal function which involves convolutions with $\Psi$ in the classes $\mathcal{S}_{n}^{\theta, j}$ and in our application it is crucial that these convolutions will be acting on functions with cancellation, namely with frequency support in annuli. Let

$$
\mathcal{M}_{n}^{\theta, j} g(x)=\sup _{\Psi \in \mathcal{S}_{n}^{\theta, j}}|\Psi * g(x)|
$$

and set, for $\tau>0$,

$$
\mathcal{K}_{\tau}^{\theta, j}(x):=\frac{2^{-j} \tau^{d}}{\left(1+\left|\ell_{\theta, j}^{-1}(\tau x)\right|\right)^{d+1}}
$$

For future reference note that

$$
|\Psi(x)| \lesssim \frac{2^{k d} 2^{-j(d+1)}}{\left(1+2^{k-2 j}|\langle x, \theta\rangle|+2^{k-j}|x-\langle x, \theta\rangle \theta|\right)^{d+3}} \lesssim \mathcal{K}_{2^{k-j}}^{\theta, j}(x) \text { if } \Psi \in \mathcal{S}_{k-j}^{\theta, j} .
$$

We use the dyadic frequency cutoff operator $P_{n}$ defined by

$$
\widehat{P_{n} f}(\xi)=\chi\left(2^{-n}|\xi|\right) \widehat{f}(\xi)
$$

where $\chi \in C^{\infty}$ is nonnegative and supported in $(5 / 8,15 / 8)$ so that $\sum_{k \in \mathbb{Z}} \chi\left(2^{-k} t\right)=1$ for all $t>0$. Set

$$
\begin{aligned}
\mathcal{W}_{q, \delta, k}^{j} g(x) & :=\left(\sup _{\theta} \mathcal{K}_{2^{k+j}}^{\theta, j} *\left|\mathcal{M}_{k-j}^{\theta, j} P_{k-j} g\right|^{q}(x)\right)^{1 / q} \\
\mathcal{W}_{q, \delta}^{j} g(x) & :=\sup _{k \in \mathbb{Z}} \mathcal{W}_{q, \delta, k}^{j} g(x) .
\end{aligned}
$$

Next, fix $s \in(1, q)$ and define the maximal operator $\mathfrak{W}_{q, \delta}$ by

$$
\mathfrak{W}_{q, \delta} w:=\sum_{1 \leq 2^{2 j}<\delta^{-1}} 2^{-2 j\left(\frac{d}{q}-1\right)}\left(M\left|M \circ \mathcal{W}_{q, \delta}^{j} w\right|^{s}\right)^{1 / s}+\delta^{\frac{d}{q}-1}\left(M\left|M \circ \mathfrak{M}_{\sqrt{\delta}} w\right|^{s}\right)^{1 / s}
$$

where $M$ is the Hardy-Littlewood maximal operator. We also recall from the statement of Theorem 2.1 the definition $\mathfrak{W}_{q} w=\sup _{0<\delta<1 / 2} \mathfrak{W}_{q, \delta} w$. 
Boundedness of the weight operators. In the proofs we will frequently use a dyadic frequency cutoff $\widetilde{P}_{n}$ which reproduces $P_{n}$ and is similarly defined. That is to say, $\widehat{\widetilde{P}_{n} f}(\xi)=$ $\widetilde{\chi}\left(2^{-n}|\xi|\right) \widehat{f}(\xi)$ where $\tilde{\chi}$ is supported in $(1 / 2,2)$ and has the property $\tilde{\chi}(s)=1$ for $s \in$ $[5 / 8,15 / 8]$. Then $P_{n} \widetilde{P}_{n}=P_{n}$.

It is obvious that the operators $\mathcal{W}_{q, \delta, k}^{j}, \mathcal{W}_{q, \delta}^{j}, \mathfrak{M}_{\sqrt{\delta}}$ are bounded on $L^{\infty}$. For the $L^{q}$ boundedness we state

Proposition 2.2. (i) For $1 \leq q \leq \infty$, the operator $\mathcal{W}_{q, \delta, k}^{j}$ satisfies

$$
\sup _{\delta, k} \sup _{\|w\|_{q} \leq 1}\left\|\mathcal{W}_{q, \delta, k}^{j} w\right\|_{q} \lesssim \begin{cases}2^{j \frac{d-2}{q}} & \text { if } 2 \leq q \leq \infty, \\ 2^{j\left(\frac{d}{q}-1\right)} & \text { if } 1 \leq q<2 .\end{cases}
$$

(ii) For $1<q \leq \infty$, the operator $\mathcal{W}_{q, \delta}^{j}$ satisfies

$$
\sup _{\|w\|_{q} \leq 1}\left\|\mathcal{W}_{q, \delta}^{j} w\right\|_{q} \lesssim \begin{cases}2^{j \frac{d-2}{q}} & \text { if } 2 \leq q \leq \infty \\ 2^{j\left(\frac{d}{q}-1\right)}\left[\log \left(\frac{1}{\delta}\right)\right]^{\frac{1}{q}-\frac{1}{2}} & \text { if } 1<q<2 .\end{cases}
$$

Moreover for $q=1$ the operator $\mathcal{W}_{1, \delta}^{j}$ maps the Hardy space $H^{1}$ to $L^{1}$ with operator norm $\lesssim 2^{j(d-1)}\left[\log \left(\frac{1}{\delta}\right)\right]^{1 / 2}$.

(iii) We also have the weak type $(q, q)$ estimate

$$
\sup _{\|w\|_{q} \leq 1}\left\|\sup _{0<\delta<1 / 2} \mathcal{W}_{\delta, q}^{j} w\right\|_{L^{q, \infty}} \lesssim \begin{cases}2^{j \frac{d-2}{q}} & \text { if } 2 \leq q<\infty \\ 2^{j\left(\frac{d}{q}-1\right)}(1+j)^{\frac{1}{q}-\frac{1}{2}} & \text { if } 1<q<2 .\end{cases}
$$

The proposition implies statements (i) and (ii) of Theorem 2.1. Clearly the operators $\mathfrak{W}_{q, \delta}$ and $\mathfrak{W}_{q}$ are bounded on $L^{\infty}$ if $q<d$. The $L^{q}$ bound for the first (main) term in (16) is immediate from Proposition 2.2 since $\frac{d-2}{q}-2\left(\frac{d}{q}-1\right)<0$ iff $q<\frac{d+2}{2}$. For the second term in (16) we use standard non-endpoint $L^{q}$ bounds for the Nikodym maximal operator (see [9], [10], 1], 27]). Namely $\mathfrak{M}_{\sqrt{\delta}}$ is bounded on $L^{q}$ with operator norm $\leq C_{\varepsilon}(\sqrt{\delta})^{1-d / q-\varepsilon}$ if $q<2$ and operator norm $\leq C_{\varepsilon}(\sqrt{\delta})^{-(d-2) / q-\varepsilon}$ if $q \geq 2$. The damping factor $\delta^{-1+d / q}$ is enough to prove $L^{q}$ boundedness for $q<\frac{d+2}{2}$. Using for example the results in [44 this final estimate can be significantly improved but any such improvement seems currently to have no impact on our result, as the main contribution to the weight operator comes from the terms $\mathcal{W}_{q, \delta}^{j}$.

Elementary convolution estimates. The following simple and standard convolution estimates will be used many times in the paper.

Lemma 2.3. (i) Let $H(x)=(1+|x|)^{-N}$ and let $N>d$. Then there is $C_{d, N}>0$ so that for all $x \in \mathbb{R}^{d}$

$$
\sup _{t \geq 1} \sup _{0 \leq s \leq 1} \int t^{d} H(t y) H(x-s y) d y \leq C_{d, N} H(x) .
$$

(ii) Let $H^{A}(x)=|\operatorname{det} A| H(A x)$. Then $H^{A} * H^{A}(x) \leq C_{d, N} H^{A}(x)$ for all $x \in \mathbb{R}^{d}$.

(iii) Let $\ell \in \mathbb{N}$, let $h_{1}, h_{2}$ be kernels with

$$
(1+|x|)^{\ell}\left|h_{1}(x)\right|+\sum_{|\alpha|=\ell}\left|\partial^{\alpha} h_{2}(x)\right| \leq H(x)
$$


and assume that $\int P(x) h_{1}(x) d x=0$ for all polynomials $P$ of degree $\leq \ell-1$. Let $h_{i}^{A}(x)=$ $|\operatorname{det} A| h_{i}(A x)$. Then for $t \geq 1$

$$
\left|t^{d} h_{1}^{A}(t \cdot) * h_{2}^{A}(x)\right| \leq C_{d, N, \ell} t^{-\ell} H^{A}(x) .
$$

Proof. Let $F_{t, s}(x)=\int t^{d} H(t y) H(x-s y) d y$ then clearly $F_{t, 0}(x) \lesssim H(x)$ and for $|x| \leq 1$ we have $F_{t, s}(x) \lesssim 1$ so that the assertion holds for $|x| \leq 1$.

If $|x| \geq 1$ then $\sup _{|s y| \leq|x| / 2} H(x-s y) \lesssim H(x)$ and $\sup _{|s y| \geq 2|x|} H(x-s y) \lesssim H(x)$, so that

$$
\int_{|s y| \notin\left[\frac{|x|}{2}, 2|x|\right]} t^{d} H(t y) H(x-s y) d y \lesssim\left\|t^{d} H(t \cdot)\right\|_{1} H(x) \lesssim H(x)
$$

for all $t>0$.

Next, if $|s y| \approx|x|$ then $t^{d} H(t y) \approx t^{d} H(t x / s)$. Letting $B_{l, s}(x)=\left\{y:|s y-x| \leq 2^{l}\right\}$ for $l \geq 0$, we have $\left|B_{l, s}(x)\right| \lesssim\left(2^{l} / s\right)^{d}$ and we may estimate (using $N>d$ )

$$
\begin{aligned}
\int_{|x| / 2 \leq|s y| \leq 2|x|} H(x-s y) & t^{d} H(t y) d y \\
& \lesssim t^{d-N} s^{N}|x|^{-N} \sum_{l \geq 0} \int_{B_{l, s}(x)} 2^{-l N} d y \lesssim t^{d-N} s^{N-d}|x|^{-N} \lesssim H(x)
\end{aligned}
$$

since $t \geq 1, s \leq 1$ and $|x| \geq 1$.

(ii) follows immediately by a change of variable. Similarly for (iii) we may reduce to the case where $A$ is the identity. Then one can use Taylor's formula and the cancellation of $h_{1}$, and (i), to estimate

$$
\begin{aligned}
\left|t^{d} h_{1}(t \cdot) * h_{2}(x)\right| & \lesssim \int_{0}^{1} \frac{(1-s)^{\ell-1}}{(\ell-1) !} \int\left|t^{d} h_{1}(t y)\langle y, \nabla\rangle^{\ell} h_{2}(x-s y)\right| d y d s \\
& \lesssim t^{-\ell} \sup _{0 \leq s \leq 1} \int t^{d} H(t y) H(x-s y) d y \lesssim t^{-\ell} H(x) .
\end{aligned}
$$

For the kernels in (11), Lemma 2.3 yields the bound

$$
\mathcal{K}_{t}^{\theta, j} * \mathcal{K}_{\tau}^{\theta, j}(x) \leq C_{d} \mathcal{K}_{\tau}^{\theta, j}(x), \quad t>\tau
$$

Proof of Proposition 2.2. We shall use many times that $\left\|\mathcal{K}_{t}^{\theta, j}\right\|_{1} \lesssim 1$, uniformly in $\theta, j, t$. Moreover,

$$
\mathcal{K}_{t}^{\theta, j}(x) \approx \mathcal{K}_{\tilde{t}}^{\tilde{\theta}, \tilde{j}}(x), \quad|\theta-\tilde{\theta}| \leq C 2^{-j}, \quad|j-\tilde{j}| \leq C, \quad C^{-1} \leq t / \tilde{t} \leq C,
$$

where the implicit constants in the equivalence depend only on $C$ and the dimension. Next, the class $\mathcal{S}_{n}^{\theta, j}$ is stable under small perturbation in the sense that given $A>0$ there is a constant $C$, depending only on $A, d$ and the parameter $\mathcal{N}$ in the definition (10), so that

$$
\Psi \in \mathcal{S}_{n}^{\theta, j} \Longrightarrow C^{-1} \Psi \in \mathcal{S}_{\tilde{n}}^{\tilde{\theta}, \tilde{j}} \text { if }|\theta-\tilde{\theta}| \leq A 2^{-j},|j-\tilde{j}| \leq A,|n-\tilde{n}| \leq A .
$$

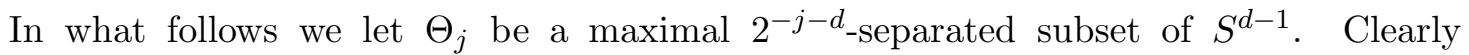
$\operatorname{card}\left(\Theta_{j}\right) \lesssim 2^{j(d-1)}$; moreover each $\tilde{\theta} \in S^{d-1}$ has distance $\leq 2^{-j}$ to at least one $\theta \in \Theta_{j}$. 
$L^{q}$-bounds for $\mathcal{W}_{q, \delta, k}^{j}$. We replace a sup in $\theta \in \Theta_{j}$ by an $\ell^{q}$ norm and interchange a summation and integration to estimate for any fixed $j, k$

$$
\begin{aligned}
& \left\|\left(\sup _{\theta} \mathcal{K}_{2^{k+j} \delta}^{\theta, j} *\left|\mathcal{M}_{k-j}^{\theta, j} P_{k-j} g\right|^{q}\right)^{1 / q}\right\|_{q} \\
& \lesssim\left(\sum_{\theta \in \Theta_{j}} \iint_{|\tilde{\theta}-\theta| \leq 2^{-j}} \sup _{2^{k+j} \delta} \mathcal{K}^{\tilde{\theta}, j}(y) \sup _{|\tilde{\theta}-\theta| \leq 2^{-j}}\left|\mathcal{M}_{k-j}^{\tilde{\theta}, j} P_{k-j} g(x-y)\right|^{q} d y d x\right)^{1 / q} \\
& \lesssim\left(\sum_{\theta \in \Theta_{j}}\left\|\mathcal{M}_{k-j}^{\theta, j} P_{k-j} g\right\|_{q}^{q}\right)^{1 / q} .
\end{aligned}
$$

For the last inequality we have used (19) and (20).

We now need a further decomposition. Let $\eta_{0}$ be supported in $(-1,1)$ so that $\eta_{0}(s)=1$ for $s \in(-1 / 2,1 / 2)$ and let

$$
\eta_{\theta}^{j, k}(\xi)=\eta_{0}\left(\sqrt{\left|2^{2 j-k}\langle\xi, \theta\rangle\right|^{2}+\left|2^{j-k}(\xi-\langle\xi, \theta\rangle \theta)\right|^{2}}\right) .
$$

For $m \geq 0$ define operators $Q_{m}^{\theta, j, k}, P_{m}^{\theta, j, k}$ as follows. For $m=0$ set

$$
\widehat{Q_{0}^{\theta, j, k}} f(\xi)=\widehat{P_{0}^{\theta, j, k}} f(\xi)=\eta_{\theta}^{j, k}(\xi) \widehat{f}(\xi)
$$

and, for $m \geq 1$, set

$$
\begin{aligned}
& \widehat{Q_{m}^{\theta, j, k}} f(\xi)=\left(\eta_{\theta}^{j, k}\left(2^{-m} \xi\right)-\eta_{\theta}^{j, k}\left(2^{-m+1} \xi\right)\right) \widehat{f}(\xi), \\
& \widehat{P_{m}^{\theta, j, k}} f(\xi)=\left(\eta_{\theta}^{j, k}\left(2^{-m} \xi\right)+\eta_{\theta}^{j, k}\left(2^{-m+1} \xi\right)\right) \widehat{f}(\xi) .
\end{aligned}
$$

Then $\sum_{m=0}^{\infty} Q_{m}^{\theta, j, k} P_{m}^{\theta, j, k}$ is the identity; moreover $Q_{m}^{\theta, j, k} P_{k-j}=0$ for $m>j+3$. Using the cancellation of $Q_{m}^{\theta, j, k}$ it is straightforward to derive the estimate

$$
\sup _{\Psi \in \mathcal{S}_{k-j}^{\theta, j}}\left|Q_{m}^{\theta, j, k} \Psi(x)\right| \leq C 2^{-2 m} \frac{2^{(k-j) d} 2^{-j}}{\left(1+2^{k-2 j}|\langle x, \theta\rangle|+2^{k-j}|x-\langle x, \theta\rangle \theta|\right)^{d+1}}
$$

from Lemma 2.3 and (12); in fact if $\mathcal{N}>d+3$ in (10) one can also get a gain of higher powers of $2^{-m}$. Now, for fixed $k, j, \theta$

$$
\begin{aligned}
\left(\sum_{\theta \in \Theta_{j}}\left\|\mathcal{M}_{k-j}^{\theta, j} P_{k-j} g\right\|_{q}^{q}\right)^{1 / q} & \lesssim\left(\sum_{\theta \in \Theta_{j}}\left\|\sup _{\Psi \in \mathcal{S}_{k-j}^{\theta, j}}\left|\sum_{0 \leq m \leq j+3}\left(Q_{m}^{\theta, j, k} \Psi\right) *\left(P_{m}^{\theta, j, k} P_{k-j} g\right)\right|\right\|_{q}^{q}\right)^{1 / q} \\
& \lesssim \sum_{0 \leq m \leq j+3} 2^{-2 m}\left(\sum_{\theta \in \Theta_{j}}\left\|P_{m}^{\theta, j, k} P_{k-j} g\right\|_{q}^{q}\right)^{1 / q}
\end{aligned}
$$

and the desired bound follows when we establish the estimate

$$
\left(\sum_{\theta \in \Theta_{j}}\left\|P_{m}^{\theta, j, k} P_{k-j} g\right\|_{q}^{q}\right)^{1 / q} \lesssim \max \left\{2^{j \frac{d-2}{q}}, 2^{j\left(\frac{d}{q}-1\right)}\right\} 2^{m / q}\|g\|_{q}, \quad 1 \leq q \leq \infty,
$$

with the usual modification for $q=\infty$. To prove (22) we notice that by interpolation we only have to verify the cases $q=1,2, \infty$. The cases $q=\infty$ and $q=1$ are immediate since the operators $P_{m}^{\theta, j, k}$ and $P_{k-j}$ have convolution kernels with uniformly bounded $L^{1}$ norms and since $\operatorname{card}\left(\Theta_{j}\right) \lesssim 2^{j(d-1)}$. For $q=2$ we use Plancherel's theorem. Write $\mathcal{F}\left[P_{m}^{\theta, j, k} P_{k-j} g\right]=a_{j, k, \theta, m} \widehat{g}$. Then the multiplier $a_{j, k, \theta, m}$ is supported in

$$
\left\{\xi: C^{-1} 2^{k-j-3} \leq|\xi| \leq C 2^{k-j+2}, \quad|\langle\xi, \theta\rangle| \leq C 2^{k-2 j+m}\right\} .
$$




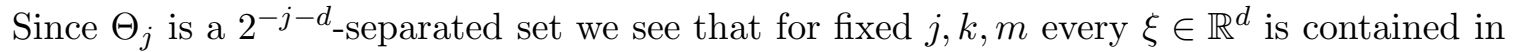
no more than $C_{d} 2^{m+j(d-2)}$ of the sets $\operatorname{supp}\left(a_{j, k, \theta, m}\right)$, which implies the bound (22).

$L^{q}$-bounds for $\mathcal{W}_{q, \delta}^{j}$. We argue as above and now replace the sup in $k \in \mathbb{Z}, \theta \in \Theta_{j}$ by an $\ell^{q}$ norm. This yields

$$
\left\|\left(\sup _{k} \sup _{\theta} \mathcal{K}_{2^{k+j} \delta}^{\theta, j} *\left|\mathcal{M}_{k-j}^{\theta, j} P_{k-j} g(x)\right|^{q}\right)^{1 / q}\right\|_{q} \lesssim\left(\sum_{k} \sum_{\theta \in \Theta_{j}}\left\|\mathcal{M}_{k-j}^{\theta, j} P_{k-j} g\right\|_{q}^{q}\right)^{1 / q} .
$$

A combination of (21) and (22) together with the use of the reproducing Littlewood-Paley cutoff $\widetilde{P}_{k-j}$ yields

$$
\begin{aligned}
& \left(\sum_{k} \sum_{\theta \in \Theta_{j}}\left\|\mathcal{M}_{k-j}^{\tilde{\theta}, j} P_{k-j} g\right\|_{q}^{q}\right)^{1 / q} \\
& \lesssim \sum_{0 \leq m \leq j+3} 2^{-2 m}\left(\sum_{k} \sum_{\theta \in \Theta_{j}}\left\|P_{m}^{\theta, j, k} P_{k-j} \widetilde{P}_{k-j} w\right\|_{q}^{q}\right)^{1 / q} \\
& \lesssim \sum_{0 \leq m \leq j+3} 2^{-m\left(2-\frac{1}{q}\right)} \max \left\{2^{j \frac{d-2}{q}}, 2^{j\left(\frac{d}{q}-1\right)}\right\}\left(\sum_{k}\left\|\widetilde{P}_{k-j} w\right\|_{q}^{q}\right)^{1 / q}
\end{aligned}
$$

and for $q \geq 2$ this is $\lesssim 2^{j(d-2) / q}\|w\|_{q}$.

Now consider the case $q<2$. By a standard linearization combined with an analytic family argument the claimed $L^{q}$ estimates can be deduced from the Hardy-space estimate and the already proven $L^{2}$ estimate. We omit the details of the interpolation argument ( $c f$. 17]). For the Hardy-space estimate we need to prove an inequality for an $L^{2}$-atom, i.e. an $L^{2}$ function $g_{\rho}$ supported on a ball $\{x:|x| \leq \rho\}$ with $\left\|g_{\rho}\right\|_{2} \leq \rho^{-d / 2}$ and $\int g_{\rho} d x=0$. It suffices to verify

$$
\left\|\mathcal{W}_{1, \delta}^{j} g_{\rho}\right\|_{1} \lesssim 2^{j(d-1)}\left[\log \left(\frac{1}{\delta}\right)\right]^{1 / 2} .
$$

By the Schwarz inequality we have

$$
\mathcal{W}_{1, \delta}^{j} g(x) \lesssim \mathcal{W}_{2, \delta}^{j} g(x)
$$

and thus by the above $L^{2}$ estimates

$$
\left\|\mathcal{W}_{1, \delta}^{j} g_{\rho}\right\|_{L^{1}(|x| \leq 2 \rho)} \lesssim \rho^{d / 2}\left\|\mathcal{W}_{2, \delta}^{j} g_{\rho}\right\|_{2} \lesssim 2^{j\left(\frac{d}{2}-1\right)} \rho^{d / 2}\left\|g_{\rho}\right\|_{2} \lesssim 2^{j\left(\frac{d}{2}-1\right)} .
$$

On the complementary set we estimate

$$
\left\|\mathcal{W}_{1, \delta}^{j} g_{\rho}\right\|_{L^{1}(|x| \geq 2 \rho)} \lesssim \sum_{k \in \mathbb{Z}}\left\|\mathcal{W}_{1, \delta, k}^{j} g_{\rho}\right\|_{L^{1}(|x| \geq 2 \rho)} .
$$

From our previous bound for $\mathcal{W}_{q, \delta, k}^{j}$ and the cancellation of the atom,

$$
\left\|\mathcal{W}_{1, \delta, k}^{j} g_{\rho}\right\|_{1} \lesssim 2^{j(d-1)}\left\|\widetilde{P}_{k-j} g_{\rho}\right\|_{1} \lesssim 2^{j(d-1)} \min \left\{1,2^{k-j} \rho\right\} .
$$

Now if $\Psi \in \mathcal{S}_{k-j}^{\theta, j}$ then by (12) with $n=k-j$, (18), and $2^{2 j} \delta \leq 1$,

$$
\mathcal{K}_{2^{k+j} \delta}^{\theta, j} *|\Psi|(x) \lesssim \mathcal{K}_{2^{\theta+j} \delta}^{\theta, j} * \mathcal{K}_{2^{\theta-j}}^{\theta, j}(x) \lesssim \frac{\left(2^{k} \delta\right)^{d} 2^{j(d-1)}}{\left(1+2^{k} \delta|\langle x, \theta\rangle|+2^{j} 2^{k} \delta|x-\langle x, \theta\rangle \theta|\right)^{d+1}} .
$$

Thus a favorable estimate holds for $2^{k} \rho \geq \delta^{-1}$, namely

$$
\left\|\mathcal{W}_{1, \delta, k}^{j} g_{\rho}\right\|_{L^{1}(|x| \geq 2 \rho)} \lesssim 2^{j(d-1)}\left(2^{k} \delta \rho\right)^{-1} \text {. }
$$


These estimates can be summed for $2^{k} \leq \rho^{-1}$ and $2^{k} \geq \delta^{-1} \rho^{-1}$. For the intermediate terms we use the $L^{1}$ bounds and the Schwarz inequality

$$
\begin{aligned}
\sum_{\rho^{-1} \leq 2^{k} \leq(\rho \delta)^{-1}}\left\|\mathcal{W}_{1, \delta, k}^{j} g_{\rho}\right\|_{1} & \lesssim 2^{j(d-1)} \sum_{\rho^{-1} \leq 2^{k} \leq(\rho \delta)^{-1}}\left\|\widetilde{P}_{k-j} g_{\rho}\right\|_{1} \\
& \lesssim 2^{j(d-1)}\left[\log \left(\frac{1}{\delta}\right)\right]^{1 / 2}\left(\sum_{k}\left\|\widetilde{P}_{k-j} g_{\rho}\right\|_{1}^{2}\right)^{1 / 2}
\end{aligned}
$$

and by Minkowski's integral inequality and the Littlewood-Paley inequality on $H^{1}$ (see e.g. [18]) we have

$$
\left(\sum_{k}\left\|\widetilde{P}_{k-j} g_{\rho}\right\|_{1}^{2}\right)^{1 / 2} \leq\left\|\left(\sum_{k}\left|\widetilde{P}_{k-j} g_{\rho}\right|^{2}\right)^{1 / 2}\right\|_{1} \lesssim\left\|g_{\rho}\right\|_{H^{1}} \lesssim 1 .
$$

Now collect the estimates and (24) is proved.

The weak type estimate. We observe the pointwise estimate

$$
\sup _{k} \mathcal{K}_{2^{k+j}{ }^{\prime}, j} *\left|\mathcal{M}_{k-j}^{\theta^{\prime}, j} P_{k-j} g\right|^{q} \lesssim M_{k}^{\theta, j}\left[\sup _{k}\left|\mathcal{M}_{k-j}^{\theta, j} P_{k-j} g\right|^{q}\right], \quad\left|\theta-\theta^{\prime}\right| \leq 2^{-j},
$$

where $M^{\theta, j}$ is the maximal operator associated with tubes of eccentricity $2^{-j}$, with the long side pointing in the direction $\theta$. For each $(\theta, j), M^{\theta, j}$ is a rescaled version of the Hardy-Littlewood maximal function and therefore satisfies the standard weak-type $(1,1)$ inequality with a bound independent of $\theta$ and $j$.

We use the continuous embedding $\ell^{q}\left(L^{q, \infty}\right) \subset L^{q, \infty}\left(\ell^{\infty}\right)$ (see e.g. Lemma 2.1 in [19]) and dominate

$$
\begin{aligned}
\left\|\sup _{0<\delta<1 / 2}\left|\mathcal{W}_{q, \delta}^{j} g\right|\right\|_{L^{q, \infty}} & \lesssim\left(\sum_{\theta \in \Theta_{j}}\left\|\left(M^{\theta, j}\left[\sup _{k}\left|\mathcal{M}_{k-j}^{\theta, j} P_{k-j} g\right|^{q}\right]\right)^{1 / q}\right\|_{L^{q, \infty}}^{q}\right)^{1 / q} \\
& \lesssim\left(\sum_{\theta \in \Theta_{j}}\left\|\sup _{k}\left|\mathcal{M}_{k-j}^{\theta, j} P_{k-j} g\right|\right\|_{L^{q}}^{q}\right)^{1 / q}
\end{aligned}
$$

and for the last inequality we have used the uniform weak-type $(1,1)$ bounds for the $M^{\theta, j}$ together with the identity $\left\|F^{q}\right\|_{L^{1, \infty}}=\|F\|_{L^{q, \infty}}^{q}$ for the usual quasinorms.

We may dominate (26) by

$$
\left(\sum_{\theta \in \Theta_{j}} \sum_{k}\left\|\mathcal{M}_{k-j}^{\theta, j} P_{k-j} g\right\|_{q}^{q}\right)^{1 / q}
$$

which for $q \geq 2$ has already been estimated by $2^{j(d-2) / q}\|g\|_{q}$. Thus the asserted estimate follows in this range.

For $1<q<2$ we claim that

$$
\left(\sum_{\theta \in \Theta_{j}}\left\|\sup _{k}\left|\mathcal{M}_{k-j}^{\theta, j} P_{k-j} g\right|\right\|_{q}^{q}\right)^{1 / q} \lesssim 2^{j\left(\frac{d}{q}-1\right)}(1+j)^{1 / q-1 / 2}\|g\|_{q} .
$$

This follows by complex interpolation from the estimate for $q=2$ already proved above and an $H^{1} \rightarrow \ell^{1}\left(L^{1}\left(\ell^{\infty}\right)\right)$ bound. Again it suffices to consider an $L^{2}$-atom $g_{\rho}$ supported on a ball of radius $\rho$ centered at the origin and we need to check

$$
\sum_{\theta \in \Theta_{j}}\left\|\sup _{k}\left|\mathcal{M}_{k-j}^{\theta, j} P_{k-j} g_{\rho}\right|\right\|_{1} \lesssim 2^{j(d-1)}(1+j)^{1 / 2}
$$


Now on the ball of radius $2 \rho$ we use an $L^{2}$ estimate and the trivial estimation $\left|\mathcal{M}_{k-j}^{\theta, j} f\right| \lesssim$ $M^{\theta, j}(f)$ to obtain

$$
\begin{aligned}
& \sum_{\theta \in \Theta_{j}}\left\|\sup _{k}\left|\mathcal{M}_{k-j}^{\theta, j} P_{k-j} g_{\rho}\right|\right\|_{L^{1}(|x| \leq 2 \rho)} \lesssim \rho^{d / 2} \sum_{\theta \in \Theta_{j}}\left\|\sup _{k}\left|\mathcal{M}_{k-j}^{\theta, j} P_{k-j} g_{\rho}\right|\right\|_{2} \\
& \lesssim \rho^{d / 2} \sum_{\theta \in \Theta_{j}}\left\|\left(\sum_{k}\left(M^{\theta, j}\left(P_{k-j} g_{\rho}\right)\right)^{2}\right)^{1 / 2}\right\|_{2} \\
& \lesssim \rho^{d / 2} \sum_{\theta \in \Theta_{j}}\left(\sum_{k}\left\|P_{k-j} g_{\rho}\right\|_{2}^{2}\right)^{1 / 2} \lesssim 2^{j(d-1)} \rho^{d / 2}\left\|g_{\rho}\right\|_{2} \lesssim 2^{j(d-1)} .
\end{aligned}
$$

For $|x| \geq 2 \rho$ we replace the sup in $k$ by the sum. By standard $L^{1}$ estimates and using the cancellation of the atom we have

$$
\left\|\mathcal{M}_{k-j}^{\theta, j} P_{k-j} g_{\rho}\right\|_{1} \lesssim\left\|P_{k-j} g_{\rho}\right\|_{1} \lesssim \min \left\{2^{k-j} \rho, 1\right\}
$$

and, using estimates of the kernels,

$$
\left\|\mathcal{M}_{k-j}^{\theta, j} P_{k-j} g_{\rho}\right\|_{L^{1}(|x| \geq 2 \rho)} \lesssim\left(2^{k-2 j} \rho\right)^{-1} \quad \text { if } 2^{k} \rho>2^{j} .
$$

Thus $\sum_{\theta \in \Theta_{j}} \sum_{2^{k} \rho \notin\left[2^{j}, 2^{2 j}\right]}\left\|\mathcal{M}_{k-j}^{\theta, j} P_{k-j} g_{\rho}\right\|_{L^{1}(|x| \geq 2 \rho)} \lesssim 2^{j(d-1)}$. Moreover, for the intermediate terms,

$$
\sum_{\theta \in \Theta_{j}} \sum_{2^{k}}\left\|\mathcal{M}_{k-j}^{\theta, j} P_{k-j} g_{\rho}\right\|_{1} \lesssim 2^{j(d-1)} \sum_{2^{k} \rho \in\left[2^{j}, 2^{2 j}\right]}\left\|P_{k-j} g_{\rho}\right\|_{1} \lesssim 2^{j(d-1)}(1+j)^{1 / 2},
$$

by the argument in (25). We combine these estimates and the $L^{1}$ bound is proved.

\section{Multipliers AND THE BILINEAR ADJOINT RESTRICTION THEOREM}

In this section we prove bilinear estimates for multiplier transformations, under suitable separation conditions. The proofs rely on

Tao's bilinear adjoint restriction theorem ([42, [24]). Let $b>1 / 2$ and $p>2+4 / d$. There exist $\varepsilon_{\circ}>0, N_{\circ} \in \mathbb{N}$ and $C$, depending on $b, p$ and $d$, that for all functions $h$ defined on $[-b, b]^{d-1}$ and satisfying

$$
\sup _{\omega \in[-b, b]^{d-1}} \max _{\substack{|\alpha| \leq N_{\circ} \\ i=1,2}}\left|\partial_{\omega}^{\alpha} h(\omega)\right| \leq \varepsilon_{0}
$$

the following holds: For all pairs of functions $\left(F_{1}, F_{2}\right)$ with $\operatorname{dist}\left(\operatorname{supp}\left(F_{1}\right), \operatorname{supp}\left(F_{2}\right)\right) \geq 1 / 2$ and $F_{i} \in L^{2}\left([-b, b]^{d-1}\right)$,

$$
\left(\int\left|\prod_{i=1,2} \int_{[-b, b]^{d-1}} F_{i}(\omega) \exp \left(\imath\left\langle x^{\prime}, \omega\right\rangle+\imath x_{d}\left(|\omega|^{2} / 2+h(\omega)\right)\right) d \omega\right|^{p / 2} d x\right)^{2 / p} \lesssim \prod_{i=1,2}\left\|F_{i}\right\|_{L^{2}} .
$$

We will need to consider families of hypersurfaces which depend on a parameter $s$ and which, for fixed $s$, are small perturbations of the paraboloid $\xi_{d}=\left|\xi^{\prime}\right|^{2} / 2$, where $\xi^{\prime}=$ $\left(\xi_{1}, \ldots, \xi_{d-1}\right)$. These lead to "elliptic" phase-functions as considered in [43], [42].

Definition. We denote by $\operatorname{Ell}\left(b, \varepsilon, N_{\circ}\right)$ the class of functions $\left(\xi^{\prime}, s\right) \mapsto \gamma\left(\xi^{\prime}, s\right)$ defined on $[-b, b]^{d-1} \times(-1,1)$ which are of the form

$$
\gamma\left(\xi^{\prime}, s\right)=\frac{\left|\xi^{\prime}\right|^{2}}{2}-s+h\left(\xi^{\prime}, s\right)
$$


with

$$
\sup _{\substack{\omega \in[-b, b]-1 \\ s \in(-1,1)}} \max _{\substack{|\alpha| \leq N_{\circ} \\ i=1,2}}\left|\partial_{\omega, s}^{\alpha} h(\omega, s)\right| \leq \varepsilon .
$$

We may and shall assume in what follows that $N_{\circ}$ is large, say $N_{\circ}>10 d$.

We now consider Fourier multipliers depending on a parameter $s \in(-1,1)$, supported in a tubular neighborhood of $\left(\xi^{\prime}, \gamma\left(\xi^{\prime}, s\right)\right)$.

Lemma 3.1. Let $p>2+4 / d, b>1 / 2$. There are $\varepsilon, N_{\circ}$, depending on $d, b$, and $p$, so that the following holds for all $\delta_{\circ}<1 / 2$.

Let $\gamma \in \operatorname{Ell}\left(b, \varepsilon, N_{\circ}\right)$. For $|s| \leq 1$ and $i=1,2$, let $a_{i}(\cdot, s)$ be multipliers, supported on $[-b, b]^{d-1}$, satisfying the conditions

$$
\begin{gathered}
\left|a_{i}(\xi, s)\right| \leq 1, \\
a_{i}(\xi, s)=0 \quad \text { if }\left|\xi_{d}-\gamma\left(\xi^{\prime}, s\right)\right| \geq \delta_{\circ},
\end{gathered}
$$

and

$$
\left(\xi^{\prime}, \xi_{d}\right) \in \operatorname{supp} a_{1}(\cdot, s), \quad\left(\widetilde{\xi}^{\prime}, \widetilde{\xi}_{d}\right) \in \operatorname{supp} a_{2}(\cdot, s) \Longrightarrow\left|\xi^{\prime}-\widetilde{\xi}^{\prime}\right| \geq 1 .
$$

Then, for all pairs of $L^{2}$ functions $\left(f_{1}, f_{2}\right)$,

$$
\left\|\int_{-1 / 2}^{1 / 2} \prod_{i=1,2} a_{i}(D, s) f_{i} d s\right\|_{p / 2} \lesssim \delta_{\circ}^{2} \prod_{i=1,2}\left\|f_{i}\right\|_{2} .
$$

Proof. For fixed $s$ we introduce coordinates

$$
\xi=\Gamma^{s}\left(\xi^{\prime}, \tau\right):=\left(\xi^{\prime}, \gamma\left(\xi^{\prime}, s\right)+\tau\right)
$$

in the Fourier integral. We then need to estimate the $L^{p / 2}$ norm of

$$
\int_{-1 / 2}^{1 / 2} \prod_{i=1,2}\left[\int_{-\delta_{\circ}}^{\delta_{\circ}} \int_{[-b, b]^{d-1}}\left[a_{i} \widehat{f}_{i}\right]\left(\Gamma^{s}\left(\xi^{i^{\prime}}, \tau^{i}\right)\right) e^{\imath\left(\left\langle x^{\prime}, \xi^{i^{\prime}}\right\rangle+x_{d}\left(\gamma\left(\xi^{i^{\prime}}, s\right)+\tau^{i}\right)\right)} d \xi^{i^{\prime}} d \tau^{i}\right] d s
$$

here we denote by $\left(\xi^{i^{\prime}}, \tau^{i}\right)$ the variables in the two different copies of $\mathbb{R}^{d}$. By Minkowski's integral inequality the $L^{p / 2}$ norm is dominated by

$$
\iiint_{\left[-\delta_{\circ}, \delta_{\circ}\right]^{2} \times\left[-\frac{1}{2}, \frac{1}{2}\right]}\left(\int\left|\prod_{i=1,2}\left[\int_{[-b, b]^{d-1}}\left[a_{i} \widehat{f}_{i}\right]\left(\Gamma^{s}\left(\xi^{i^{\prime}}, \tau^{i}\right)\right) e^{\imath\left(\left\langle x^{\prime}, \xi^{i^{\prime}}\right\rangle+x_{d} \gamma\left(\xi^{i}, s\right)\right)} d \xi^{i}\right]\right|^{p / 2} d x\right)^{2 / p} d s d \tau^{1} d \tau^{2} .
$$

By the bilinear adjoint restriction theorem and the boundedness of $a_{i}$ this is estimated by

$$
\iiint_{\left[-\delta_{\circ}, \delta_{0}\right]^{2} \times\left[-\frac{1}{2}, \frac{1}{2}\right]} \prod_{i=1,2}\left(\int_{[-b, b]}\left|\widehat{f}_{i}\left(\Gamma^{s}\left(\xi^{i^{\prime}}, \tau^{i}\right)\right)\right|^{2} d \xi^{i^{\prime}}\right)^{1 / 2} d s d \tau^{1} d \tau^{2} .
$$

We apply the Schwarz inequality in the $s$ variable. Then for fixed $\tau^{1}, \tau^{2}$, we change variables $\left(\xi^{\prime}, s\right) \mapsto \xi=\left(\xi^{\prime}, \gamma\left(\xi^{\prime}, s\right)+\tau^{i}\right)$, using that $\partial_{s} \gamma\left(\xi^{\prime}, s\right)=1+O(\varepsilon)$. Thus the last displayed 
expression is estimated by

$$
\begin{aligned}
\iint_{\left[-\delta_{\circ}, \delta_{\circ}\right]^{2}} \prod_{i=1,2}\left(\int_{[-b, b]^{d-1} \times[-1,1]} \mid \widehat{f}\left(\xi^{i^{\prime}}, \gamma\left(\xi^{i^{\prime}}, s\right)+\right.\right. & \left.\left.\tau^{i}\right)\left.\right|^{2} d s d \xi^{i^{\prime}}\right)^{1 / 2} d \tau^{1} d \tau^{2} \\
& \lesssim \iint_{\left[-\delta_{\circ}, \delta_{\circ}\right]^{2}} \prod_{i=1,2}\left\|\widehat{f}_{i}\right\|_{2} d \tau^{1} d \tau^{2} \lesssim \delta_{\circ}^{2} \prod_{i=1,2}\left\|f_{i}\right\|_{2} .
\end{aligned}
$$

In what follows we will use the notation $\prod_{i=1,2}^{*}\left[\mathcal{E}_{i}\right]=\mathcal{E}_{1} \overline{\mathcal{E}_{2}}$ for products involving a complex conjugate.

Proposition 3.2. Let $q \in\left[1, \frac{d+2}{2}\right)$ and $b>1 / 2$. There are $\varepsilon, N_{\circ}$, depending on $d, b$ and $q$, so that the following holds for all $\delta_{\circ}<1 / 2$.

Let $\gamma \in \operatorname{Ell}\left(b, \varepsilon, N_{\circ}\right)$. For $|s| \leq 1$ and $i=1,2$, let $a_{i}(\cdot, s)$ be multipliers, supported on $[-b, b]^{d-1}$, satisfying the conditions

$$
\begin{gathered}
\left|\partial_{\xi}^{\alpha} a_{i}(\xi, s)\right| \leq \delta_{\circ}^{-|\alpha|}, \quad|\alpha| \leq d+2, \\
a_{i}(\xi, s)=0 \quad \text { if }\left|\xi_{d}-\gamma\left(\xi^{\prime}, s\right)\right| \geq \delta_{\circ},
\end{gathered}
$$

and

$$
\left(\xi^{\prime}, \xi_{d}\right) \in \operatorname{supp} a_{1}(\cdot, s), \quad\left(\widetilde{\xi}^{\prime}, \widetilde{\xi}_{d}\right) \in \operatorname{supp} a_{2}(\cdot, s) \Longrightarrow\left|\xi^{\prime}-\widetilde{\xi}^{\prime}\right| \geq 1
$$

Then

$$
\begin{aligned}
& \left|\int_{\mathbb{R}^{d}} \int_{-1 / 2}^{1 / 2} \prod_{i=1,2}^{*}\left[a_{i}(D, s) f_{i}(x)\right] w(x) d x d s\right| \\
& \leq C_{N} \delta_{\circ}^{2} \prod_{i=1,2}\left(\int\left|f_{i}(x)\right|^{2}\left(\int \frac{|w(x-y)|^{q}}{\left(1+\delta_{\circ}|y|\right)^{(d+1) q}} d y\right)^{1 / q} d x\right)^{1 / 2} .
\end{aligned}
$$

Proof. We dyadically decompose the kernel of the convolution operators. Let $\rho_{0}$ be a $C_{c}^{\infty}(\mathbb{R})$ function supported on $(-1,1)$ and equal to one on $[-1 / 2,1 / 2]$ and define, for $x \in \mathbb{R}^{d}$,

$$
\Phi_{0}(x)=\rho_{0}\left(\delta_{\circ}|x|\right), \quad \Phi_{j}(x)=\rho_{0}\left(2^{-j} \delta_{\circ}|x|\right)-\rho_{0}\left(2^{1-j} \delta_{\circ}|x|\right), \quad j \geq 1 .
$$

Then the $\left\{\Phi_{j}\right\}_{j=0}^{\infty}$ form a radial partition of unity. We thus need to bound the sum

$$
\sum_{j_{1}, j_{2} \geq 0}\left|\int_{\mathbb{R}^{d}} \int_{-1 / 2}^{1 / 2} \prod_{i=1,2}^{*}\left[\int a_{i}\left(D-\eta^{i}, s\right) f_{i}(x) \widehat{\Phi}_{j_{i}}\left(\eta^{i}\right) d \eta^{i}\right] w(x) d x d s\right| .
$$

By symmetry considerations it suffices to consider the terms with $0 \leq j_{1} \leq j_{2}$. The desired estimate then follows if we can show that

$$
\begin{aligned}
& \mid \int_{\mathbb{R}^{d}} \int_{-1 / 2}^{1 / 2} \prod_{i=1,2}^{*} {\left[\int a_{i}\left(D-\eta^{i}, s\right) f_{i}(x) \widehat{\Phi}_{j_{i}}\left(\eta^{i}\right) d \eta^{i}\right] d s w(x) d x \mid \lesssim } \\
& C(L) \delta_{\circ}^{2} 2^{-j_{2} L} \prod_{i=1,2}\left(\int\left|f_{i}(x)\right|^{2}\left(\int_{|y| \leq C 2^{j_{2} \delta_{\circ}^{-1}}}|w(x+y)|^{q} d y\right)^{1 / q} d x\right)^{1 / 2}
\end{aligned}
$$

for $0 \leq j_{1} \leq j_{2}, L \leq d+2$. We shall first verify this inequality for $L=0$ and then provide the modification for $0<L \leq d+2$. 
We now form a grid $\mathfrak{Q}\left(j_{2}\right)$ of dyadic cubes of sidelength $2^{j_{2}} \delta_{\circ}^{-1}$. For every $Q \in \mathfrak{Q}\left(j_{2}\right)$ let $Q^{*}$ be the double cube with same center as $Q$. By the support properties of the kernels, and $j_{1} \leq j_{2}$, we have

$$
\int a_{i}\left(D-\eta^{i}, s\right)\left[f_{i} \chi_{\mathbb{R}^{d} \backslash Q^{*}}\right](x) \widehat{\Phi}_{j_{i}}\left(\eta_{i}\right) d \eta_{i}=0 \quad \text { if } x \in Q, \quad Q \in \mathfrak{Q}\left(j_{2}\right), \quad i=1,2 .
$$

Thus the left hand side of (34) is equal to

$$
\left|\sum_{Q \in \mathcal{Q}\left(j_{2}\right)} \int_{Q} \int_{-1 / 2}^{1 / 2} \prod_{i=1,2}^{*}\left[\int a_{i}\left(D-\eta^{i}, s\right)\left[\chi_{Q^{*}} f_{i}\right](x) \widehat{\Phi}_{j_{i}}\left(\eta^{i}\right) d \eta^{i}\right] d s w(x) d x\right| .
$$

We use the formula $m(D-\eta) f=\operatorname{Mod}_{\eta} m(D)\left[\operatorname{Mod}_{-\eta} f\right]$ where $\operatorname{Mod}_{\eta} g(x)=g(x) e^{\imath\langle x, \eta\rangle}$. In order to obtain (34) for $L=0$ (which is efficient for $j_{2}=0$ ) we use Hölder's inequality to estimate (35) by

$$
\iint\left|\widehat{\Phi}_{j_{1}}\left(\eta^{1}\right) \widehat{\Phi}_{j_{2}}\left(\eta^{2}\right)\right| \sum_{Q \in \mathfrak{Q}\left(j_{2}\right)}\left\|\int_{-1 / 2}^{1 / 2} \prod_{i=1,2}^{*}\left[a_{i}(D, s) \operatorname{Mod}_{-\eta^{i}}\left[\chi_{Q^{*}} f_{i}\right]\right]\right\|\left\|_{q^{\prime}}\right\| w \chi_{Q} \|_{q} d \eta^{1} d \eta^{2}
$$

Now

$$
\begin{aligned}
& \left\|\int_{-1 / 2}^{1 / 2} \prod_{i=1,2}^{*}\left[a_{i}(D, s) \operatorname{Mod}_{-\eta^{i}}\left[\chi_{Q^{*}} f_{i}\right]\right] d s\right\|_{q^{\prime}} \\
\leq & \int_{-1 / 2}^{1 / 2}\left\|\prod_{i=1,2}^{*}\left[a_{i}(D, s) \operatorname{Mod}_{-\eta^{i}}\left[\chi_{Q^{*}} f_{i}\right]\right]\right\|_{q^{\prime}} d s \\
= & \int_{-1 / 2}^{1 / 2}\left\|\prod_{i=1,2} a_{i}(D, s) \operatorname{Mod}_{-\eta^{i}}\left[\chi_{Q^{*}} f_{i}\right]\right\|_{q^{\prime}} d s .
\end{aligned}
$$

By our assumption on $q$ we have $q^{\prime}=p / 2$ for some $p>2+4 / d$ and therefore we can use Lemma 3.1 to bound the last displayed expression by

$$
\delta_{\circ}^{2} \prod_{i=1,2}\left\|f_{i} \chi_{Q^{*}}\right\|_{2}
$$

Since the $L^{1}$ norms of $\widehat{\Phi}_{j_{1}}$ are $O(1)$, uniformly in $\delta_{\circ}$, we have

$$
\begin{aligned}
(35) & \lesssim \delta_{\circ}^{2} \sum_{Q \in \mathfrak{Q}\left(j_{2}\right)}\left\|f_{1} \chi_{Q^{*}}\right\|_{2}\left\|f_{2} \chi_{Q^{*}}\right\|_{2}\left\|w \chi_{Q}\right\|_{q} \\
& \lesssim \delta_{\circ}^{2} \prod_{i=1,2}\left(\sum_{Q \in \mathfrak{Q}\left(j_{2}\right)}\left\|f_{i} \chi_{Q^{*}}\right\|_{2}^{2}\left\|w \chi_{Q}\right\|_{q}\right)^{1 / 2} \\
& \lesssim \delta_{\circ}^{2} \prod_{i=1,2}\left(\int_{Q^{*}}\left|f_{i}(x)\right|^{2}\left(\int_{|y| \leq C 2^{j_{2}} \delta_{\circ}^{-1}}|w(x+y)|^{q} d y\right)^{1 / q} d x\right)^{1 / 2}
\end{aligned}
$$

which yields (34) for $L=0$.

We now turn to the case $L \leq d+2$ where we need to improve the above estimate by a factor of $C(L) 2^{-j_{2} L}$. We expand the convolution $\int a_{2}(\xi-\eta, s) \widehat{\Phi}_{j_{2}}(\eta) d \eta$ by a Taylor expansion about $\eta=0$. Since $\Phi_{j_{2}}$ vanishes in a neighborhood of the origin the integrals 
$\int \widehat{\Phi}_{j_{2}}(\eta) P(\eta) d \eta$ are zero for any polynomial $P$. Thus only the integral remainder term in the Taylor expansion survives and we obtain

$$
a_{2}(\cdot, s) * \widehat{\Phi}_{j_{2}}(\xi)=\int_{0}^{1} \frac{(1-\sigma)^{L-1}}{(L-1) !} \int \widehat{\Phi}_{j_{2}}(\eta)\left\langle-\eta, \nabla_{\xi}\right\rangle^{L} a_{2}(\xi-\sigma \eta, s) d \eta d \sigma .
$$

We repeat the above argument in which we now have to bound

$$
\begin{aligned}
\int_{\eta^{1}} & \int_{\eta^{2}} \int_{0}^{1}\left|\widehat{\Phi}_{j_{1}}\left(\eta^{1}\right) d \eta^{1}\right|\left|\widehat{\Phi}_{j_{2}}\left(\eta^{2}\right) d \eta^{2}\right| \sum_{Q \in \mathcal{Q}\left(j_{2}\right)} \int_{x \in Q}|w(x)| \times \\
& \left|\int_{-1 / 2}^{1 / 2} a_{1}\left(D-\eta^{1}, s\right)\left[\chi_{Q^{*}} f_{1}\right](x) \overline{\left\langle-\eta^{2}, \nabla\right\rangle^{L} a_{2}\left(D-\sigma \eta^{2}, s\right)\left[\chi_{Q^{*}} f_{2}\right](x)} d s\right| d x d \sigma d \eta^{1} d \eta^{2}
\end{aligned}
$$

in place of (35). In the estimate we may replace $\left\langle-\eta^{2}, \nabla\right\rangle^{L}$ with $\left(\eta^{2}\right)^{\alpha} \partial_{\xi}^{\alpha}$, for any multiindex $\alpha$ with $|\alpha|=L$. As above we continue with Hölder's inequality, and this time Lemma 3.1 and the differentiability assumptions on $a_{2}$ yield for $|\alpha|=L$

$$
\begin{aligned}
\int_{0}^{1} \| \int_{-1 / 2}^{1 / 2} a_{1}(D, s)\left[\chi_{Q^{*}} \operatorname{Mod}_{-\eta^{1}} f_{1}\right] \overline{\partial_{\xi}^{\alpha} a_{2}(D, s) \operatorname{Mod}_{-\sigma \eta^{2}}\left[\chi_{Q^{*}} f_{2}\right]} & d s \|_{q^{\prime}} d \sigma \\
& \lesssim \delta_{\circ}^{2-L} \prod_{i=1,2}\left\|f_{i} \chi_{Q^{*}}\right\|_{2} .
\end{aligned}
$$

The loss of $\delta_{\circ}^{-L}$ in the previous formula is (more than) mitigated by

$$
\iint\left|\widehat{\Phi}_{j_{1}}\left(\eta^{1}\right)\left(\eta^{2}\right)^{\alpha} \widehat{\Phi}_{j_{2}}\left(\eta^{2}\right)\right| d \eta^{1} d \eta^{2} \lesssim 2^{-j_{2} L} \delta_{\circ}^{L}, \quad|\alpha|=L .
$$

Thus the above argument yields (34) for also for $0<L \leq d+2$.

\section{Proof of the Weighted inequality}

In this section we prove inequality (7) of Theorem 2.1. We mainly focus on a local inequality (with $t$-interval $[1,2]$ ) which for later application we formulate for slightly more general multipliers. Instead of $S_{t}^{\delta}$ we consider operators $\mathcal{S}_{t}^{\delta}$ defined by

$$
\widehat{\mathcal{S}_{t}^{\delta} f}(\xi)=\phi\left(\delta^{-1}\left(1-\frac{|\xi|^{2}}{t^{2}}\right) \beta(\xi, t)\right) \widehat{f}(\xi)
$$

where $\phi$ is as in (5), $\beta$ is a nonvanishing $C^{\infty}$ function on the set of $(\xi, t)$ with $1 / 2<t<5 / 2$ and $1 / 2 \leq|\xi| \leq 4$. Of course $\beta(\xi, t)=1$ in Theorem 2.1.

Theorem 4.1. Let $d \geq 2$ and $q \in\left[1, \frac{d+2}{2}\right)$. Then, for $0<\delta<1 / 2$,

$$
\int_{\mathbb{R}^{d}} \int_{1}^{2}\left|\mathcal{S}_{t}^{\delta} f(x)\right|^{2} \frac{d t}{t} w(x) d x \lesssim \delta^{2-d / q} \int_{\mathbb{R}^{d}}|f(x)|^{2} W w(x) d x
$$

with

$$
W w(x)=\sum_{1 \leq 2^{2 j}<\delta^{-1}} 2^{-2 j\left(\frac{d}{q}-1\right)} M \circ \mathcal{W}_{q, \delta, 0}^{j} w(x)+\delta^{\frac{d}{q}-1} M \circ \mathfrak{M}_{\sqrt{\delta}} w(x)
$$


We now show that Theorem 4.1] (with $\beta \equiv 1$ ) implies assertion (77) of Theorem 2.1. Let $W_{k} w(x)=W\left[w\left(2^{-k} \cdot\right)\right]\left(2^{k} x\right)$. By (37) and rescaling we see that

$$
\begin{aligned}
& \int_{\mathbb{R}^{d}} \int_{0}^{\infty}\left|S_{t}^{\delta} f(x)\right|^{2} \frac{d t}{t} w(x) d x \\
= & \sum_{k \in \mathbb{Z}} \int_{\mathbb{R}^{d}} \int_{1}^{2}\left|S_{2^{k} s}^{\delta} \sum_{i=-2}^{2} P_{k+i} f(x)\right|^{2} \frac{d s}{s} w(x) d x \\
\lesssim & \delta^{2-d / q} \sum_{k \in \mathbb{Z}} \sum_{i=-2}^{2} \int_{\mathbb{R}^{d}} \int_{1}^{2}\left|P_{k+i} f(x)\right|^{2} W_{k} w(x) d x \frac{d s}{s} \\
\lesssim & \delta^{2-d / q} \int_{\mathbb{R}^{d}} \int_{1}^{2}|f(x)|^{2} M\left(\sup _{k}\left|W_{k} w\right|^{s}\right)^{1 / s}(x) d x
\end{aligned}
$$

and the last inequality is a consequence of Coifman's improvement of the Córdoba-Fefferman weighted norm inequality for singular integrals (see for example [18, p. 417]). Now $M\left(\sup _{k}\left|W_{k} w\right|^{s}\right)^{1 / s}(x) \lesssim \mathfrak{W}_{q, \delta} w$, by Minkowski's inequality (cf. (16) $)$.

Theorem 4.1 implies the following sharp $L^{p}$ results, by a duality argument, the boundedness results of Proposition 2.2 and the Marcinkiewicz interpolation theorem.

Corollary 4.2. Let $d \geq 2$ and $p \in\left(\frac{2(d+2)}{d}, \infty\right)$. Then, for $0<\delta<1 / 2$,

$$
\left\|\left(\int_{1}^{2}\left|\mathcal{S}_{t}^{\delta} f(x)\right|^{2} \frac{d t}{t}\right)^{1 / 2}\right\|_{p} \lesssim \delta^{1-d\left(\frac{1}{2}-\frac{1}{p}\right)}\|f\|_{p}
$$

Moreover if $\beta \equiv 1$ and $S_{t}^{\delta}$ is as in (6) then

$$
\left\|\left(\int_{0}^{\infty}\left|S_{t}^{\delta} f(x)\right|^{2} \frac{d t}{t}\right)^{1 / 2}\right\|_{p} \lesssim \delta^{1-d\left(\frac{1}{2}-\frac{1}{p}\right)}\|f\|_{p} .
$$

The remainder of this section is devoted to the proof of Theorem 4.1 .

Preliminary considerations. We begin with a rescaled variant of Proposition 3.2. Such rescaling arguments have been used in [43, [42, [23] and elsewhere. In what follows fix a function $\zeta \in C^{\infty}\left(\mathbb{R}^{d}\right)$ supported in $\{y:|y| \leq 1 / 8\}$ and define a convolution operator with homogeneous multiplier by

$$
\widehat{Q^{\theta, j} f}=\zeta\left(2^{j}\left(\frac{\xi}{|\xi|}-\theta\right)\right.
$$

In order to reduce estimates to Proposition 3.2 by rescaling we will need to localize all multipliers to a narrow sector $\left\{\xi:\left|\frac{\xi}{|\xi|}-u\right| \leq \varepsilon_{1}\right\}$ where $u$ is a unit vector and $\varepsilon_{1}$ is a small constant.

Lemma 4.3. Given $C>1$ there are small $\varepsilon_{1}, \varepsilon_{2} \in(0,1 / 8)$ depending on $q, d$ and $C$ and the function $\beta$ so that the following statement holds for $C 2^{-j}<\varepsilon_{1}$ and $2^{2 j} \delta<\varepsilon_{2}$.

Let $\psi$ be supported in a ball of radius $\varepsilon_{1}$ contained in $\{\xi: 1 / 4 \leq|\xi| \leq 4\}$ such that $\left\|\partial^{\alpha} \psi\right\|_{\infty} \leq 1$ for $|\alpha| \leq d+2$, let $\theta_{1}, \theta_{2} \in S^{d-1}$ be such that $2^{-j-1} \leq\left|\theta_{1}-\theta_{2}\right| \leq C 2^{-j}$, let $t_{0} \in[1,2]$ and $J$ be an interval of length $2^{-2 j-2}$ containing $t_{0}$. Then

$$
\begin{aligned}
\left|\iint_{J} \mathcal{S}_{t}^{\delta} Q^{\theta_{1}, j} \psi(D) f_{1}(x) \overline{\mathcal{S}_{t}^{\delta} Q^{\theta_{2}, j} \psi(D) f_{2}(x)} \frac{d t}{t} w(x) d x\right| \\
\quad \lesssim \delta\left(2^{2 j} \delta\right)^{1-d / q} \prod_{i=1,2}\left(\int\left|f_{i}(x)\right|^{2}\left(\mathcal{K}_{2^{j} \delta}^{\theta_{i}, j} *|w|^{q}(x)\right)^{1 / q} d x\right)^{1 / 2} .
\end{aligned}
$$


Proof. Set $\vartheta=\frac{\theta_{1}+\theta_{2}}{\left|\theta_{1}+\theta_{2}\right|}$ and $e_{d}=(0, \ldots, 0,1)$. Take $R_{\vartheta}$ to be a rotation satisfying $R_{\vartheta} e_{d}=\vartheta$ and let it act on functions by $\mathcal{R}_{\vartheta} f(y)=f\left(R_{\vartheta} y\right)$. Let $\mathcal{A}_{j} f(y)=f\left(A_{j} y\right)$, where $A_{j} y=$ $\left(2^{j} y_{1}, \ldots, 2^{j} y_{d-1},-2^{2 j} y_{d}\right)$. Finally we set $\mathcal{D}_{t_{0}} f(y)=f\left(t_{0} y\right)$ and, as before, the modulation $\operatorname{Mod}_{a}$ is defined by $\operatorname{Mod}_{a} g(x)=g(x) e^{\imath\langle x, a\rangle}$.

For fixed $\delta, j$ and $i=1,2$, the multiplier for $S_{t}^{\delta} Q^{\theta_{i}, j} \psi(D)$ is given by

$$
m_{\theta_{i}}(\xi, t)=\psi(\xi) \phi\left(\delta^{-1}\left(1-\frac{|\xi|^{2}}{t^{2}}\right) \beta(\xi, t)\right) \zeta\left(2^{j}\left(\frac{\xi}{|\xi|}-\theta_{i}\right)\right) .
$$

Let $\Xi_{\vartheta}(\eta)=t_{0} R_{\vartheta}\left(e_{d}+A_{-j} \eta\right)$ and $t(s)=t_{0}\left(1+2^{-2 j} s\right)$. A rescaled multiplier depending on the parameter $s=2^{2 j}\left(t_{0}^{-1} t-1\right) \in[-1 / 2,1 / 2]$ is defined by

$$
\begin{aligned}
M_{\theta_{i}}(\eta, s) & :=m_{\theta_{i}}\left(\Xi_{\vartheta}(\eta), t(s)\right) \\
& =\phi\left(\delta^{-1}\left(1-\frac{\left|e_{d}+A_{-j} \eta\right|^{2}}{\left(1+2^{-2 j} s\right)^{2}}\right) \beta\left(\Xi_{\vartheta}(\eta), t(s)\right)\right) \zeta\left(2^{j} R_{\vartheta}\left[\frac{e_{d}+A_{-j} \eta}{\left|e_{d}+A_{-j} \eta\right|}-R_{\vartheta}^{-1} \theta_{i}\right]\right) \psi\left(\Xi_{\vartheta}(\eta)\right) .
\end{aligned}
$$

Now compute

$$
\begin{aligned}
1-\frac{\left|e_{d}+A_{-j} \eta\right|^{2}}{\left(1+2^{-2 j} s\right)^{2}} & =2^{1-2 j}\left(\frac{s\left(1+2^{-2 j-1} s\right)+\eta_{d}-\left|\eta^{\prime}\right|^{2} / 2-\eta_{d}^{2} 2^{-2 j-1}}{\left(1+2^{-2 j} s\right)^{2}}\right) \\
& =2^{1-2 j}\left(s+\eta_{d}-\frac{\left|\eta^{\prime}\right|^{2}}{2}\right)+2^{-4 j} r_{j}(\eta, s),
\end{aligned}
$$

where $r_{j}$ is a quadratic polynomial in $\eta$ with coefficients uniformly bounded in $s, j$. Moreover the supports of the functions $\zeta\left(2^{j} R_{\vartheta}\left[\frac{e_{d}+A_{-j} \eta}{\left|e_{d}+A_{-j} \eta\right|}-R_{\vartheta}^{-1} \theta_{i}\right]\right), i=1,2$, are uniformly separated and these functions have derivatives with bounds uniform in $j$.

Now let $b=10 d C$, and set $M=1+\sum_{|\alpha| \leq 2} \max _{|\xi| \leq b} \max _{\mid s \leq 1}\left|\partial_{\eta}^{\alpha} r_{j}(\eta, s)\right|$, for the error terms $r_{j}$ in (39). Let $\varepsilon$ be as in Proposition [3.2, and choose $\varepsilon_{1}$ small compared to $(2 d)^{-1}(\varepsilon / M)^{1 / 2}$. By the assumed separation property we have

$$
\mathcal{S}_{t}^{\delta} Q^{\theta_{1}, j} \psi(D) f_{1} \overline{\mathcal{S}_{t}^{\delta} Q^{\theta_{2}, j} \psi(D) f_{2}} \equiv 0, \quad 2^{-j} \geq d \varepsilon_{1}
$$

For the relevant complementary range we have $2^{-2 j} M<\varepsilon$ so that the functions $\left(\eta^{\prime}, s\right) \mapsto$ $\left|\eta^{\prime}\right|^{2} / 2-s+2^{-2 j-1} r_{j}\left(\eta^{\prime}, s\right)$ belong to $\operatorname{Ell}\left(b, \varepsilon, N_{\circ}\right)$. Since $\beta$ is smooth and satisfies an inequality $2 \varepsilon_{2} \leq|\beta(\xi, t)| \leq\left(2 \varepsilon_{2}\right)^{-1}$ for $1 / 4 \leq|\xi| \leq 4$, the formula (39) allows us to apply Proposition 3.2 with $a_{i}(D, s)=M_{\theta_{i}}(D, s)\left(1+2^{-2 j} s\right)^{-1 / 2}$ and $\delta_{\circ}=\left(2 \varepsilon_{2}\right)^{-1} \delta 2^{2 j}<1 / 2$.

We obtain

$$
\begin{aligned}
\mid \int_{\mathbb{R}^{d}} \int_{-1 / 2}^{1 / 2} \prod_{i=1,2}^{*}\left[M_{\theta_{i}}(D, s) f_{i}(x)\right] & w(x) d x \frac{d s}{1+2^{-2 j} s} \mid \\
& \lesssim\left(2^{2 j} \delta\right)^{2} \prod_{i=1,2}\left(\int\left|f_{i}(x)\right|^{2}\left(2^{2 j} \delta\right)^{-d / q} \mathcal{V}_{2^{2 j} \delta} w(x) d x\right)^{1 / 2}
\end{aligned}
$$

where

$$
\mathcal{V}_{\delta_{\circ}} w(x)=\left(\int \frac{\delta_{\circ}^{d}|w(x-y)|^{q}}{\left(1+\delta_{\circ}|y|\right)^{(d+1) q}} d y\right)^{1 / q} .
$$

Now, with $t=t_{0}\left(1+2^{-2 j} s\right)$, we have $m_{\theta_{i}}(\xi, t)=M_{\theta_{i}}\left(A_{j}\left(R_{\vartheta}^{-1} t_{0}^{-1} \xi-e_{d}\right), s\right)$, so that

$$
\mathcal{S}_{t}^{\delta} Q^{\theta_{i}, j} \psi(D) f_{i}(x)=\mathcal{D}_{t_{0}} \mathcal{R}_{\vartheta}^{-1} \operatorname{Mod}_{e_{d}} \mathcal{A}_{-j} M_{\theta_{i}}(D, s) g_{i}(x) \text { with } g_{i}=\mathcal{A}_{j} \operatorname{Mod}_{-e_{d}} \mathcal{R}_{\vartheta} \mathcal{D}_{t_{0}}^{-1} f_{i} \text {. }
$$


This leads to

$$
\begin{aligned}
& \left|\iint_{I_{j, T}} \prod_{i=1,2}^{*}\left[\mathcal{S}_{t}^{\delta} Q^{\theta_{i}, j} \psi(D) f_{i}(x)\right] \frac{d t}{t} w(x) d x\right| \\
= & 2^{-2 j}\left|\iint_{-1 / 2}^{1 / 2} \prod_{i=1,2}^{*}\left[\mathcal{D}_{t_{0}} \mathcal{R}_{\vartheta}^{-1} \operatorname{Mod}_{e_{d}} \mathcal{A}_{-j} M_{\theta_{i}}(D, s) g_{i}(x)\right] \frac{d s}{1+2^{-2 j} s} w(x) d x\right| \\
\lesssim & 2^{-2 j}\left(2^{2 j} \delta\right)^{2} \prod_{i=1,2}\left(\int\left|f_{i}(x)\right|^{2}\left(2^{2 j} \delta\right)^{-d / q} \mathcal{D}_{t_{0}} \mathcal{R}_{\vartheta}^{-1} \mathcal{A}_{j}\left[\mathcal{V}_{2^{2 j} \delta}\left(\mathcal{A}_{j} \mathcal{R}_{\vartheta} \mathcal{D}_{t_{0}}^{-1} w\right)\right](x) d x\right)^{1 / 2} \\
\lesssim & 2^{2 j(1-d / q)} \delta^{2-d / q} \prod_{i=1,2}\left(\int\left|f_{i}(x)\right|^{2}\left(\mathcal{K}_{2^{j} t_{0} \delta}^{\vartheta} *|w|^{q}(x)\right)^{1 / q} d x\right)^{1 / 2} .
\end{aligned}
$$

The assertion now follows from $\mathcal{K}_{2^{j} t_{0} \delta}^{\vartheta}(x) \approx \mathcal{K}_{2^{j} \delta}^{\theta_{i}, j}(x)$ since $t_{0} \in[1,2]$ and $\left|\vartheta-\theta_{i}\right| \lesssim 2^{-j}$ for $i=1,2$.

A version of the following lemma is originally due to Carleson (unpublished); slightly different forms can be found in [11], 30] and [34]. For the sake of completeness we include the proof.

Lemma 4.4. Let $A$ be an invertible linear transformation and $A^{t}$ its transpose. Suppose that $\left\{m_{k}\right\}_{k \in \mathbb{N}}$ have disjoint supports. Then for $s \geq 0$ and almost every $x \in \mathbb{R}^{d}$

$$
\left(\sum_{k}\left|\mathcal{F}^{-1}\left[m_{k}\left(A^{t} \cdot\right) \widehat{f}\right](x)\right|^{2}\right)^{1 / 2} \leq C \sup _{k}\left\|m_{k}\right\|_{L_{s}^{2}}\left(\int \frac{\operatorname{det}\left(A^{-1}\right)}{\left(1+\left|A^{-1} y\right|^{2}\right)^{s}}|f(x-y)|^{2} d y\right)^{1 / 2} .
$$

Proof. We can assume that $m_{k}=0$ for all but finitely many $k$. Since $\mathcal{F}^{-1}\left[m_{k}\left(A^{t} \cdot\right) \widehat{f}\right](x)=$ $\mathcal{F}^{-1}\left[m_{k} \widehat{f(A \cdot)}\right]\left(A^{-1} x\right)$ we can reduce to the case where $A$ is the identity transformation. Also, using an analytic interpolation argument, we may assume that $s \in \mathbb{N} \cup\{0\}$.

Let $g_{k}=\mathcal{F}^{-1}\left[m_{k}\right]$. Then $\sum_{k}\left|\mathcal{F}^{-1}\left[m_{k} \widehat{f}\right](x)\right|^{2}=\sup _{\|a\|_{\ell^{2}\left(\mathbb{Z}^{d}\right)} \leq 1}\left|\sum_{k} a_{k} g_{k} * f(x)\right|^{2}$. Now, for each fixed $a \in \ell^{2}\left(\mathbb{Z}^{d}\right)$, we apply the Schwarz inequality in the convolution integral and then Plancherel's theorem to obtain

$$
\left|\sum_{k} a_{k} g_{k} * f(x)\right| \lesssim\left\|\sum_{k} a_{k} m_{k}\right\|_{L_{s}^{2}}\left(\int \frac{|f(x-y)|^{2}}{\left(1+|y|^{2}\right)^{s}} d y\right)^{1 / 2} .
$$

Thus, we are done as for $s \in \mathbb{N} \cup\{0\}$,

$$
\left\|\sum_{k} a_{k} m_{k}\right\|_{L_{s}^{2}}^{2} \lesssim \sum_{|\alpha| \leq s}\left\|\sum_{k} a_{k} D^{\alpha} m_{k}\right\|_{L^{2}}^{2} \lesssim\|a\|_{\ell^{2}}^{2} \sup _{k}\left\|m_{k}\right\|_{L_{s}^{2}}^{2}
$$

by the disjointness of the supports.

Some reductions. We remark that it suffices to prove Theorem 4.1 only for very small values of $\delta$, as by straightforward estimation

$$
\int_{\mathbb{R}^{d}} \int_{1}^{2}\left|\mathcal{S}_{t}^{\delta} f(x)\right|^{2} \frac{d t}{t} w(x) d x \lesssim \delta^{-C} \int_{\mathbb{R}^{d}}|f(x)|^{2} M w(x) d x
$$

for a suitable power $C>0$, and clearly $M w \lesssim \mathfrak{M}_{\sqrt{\delta}} w$. In particular we may assume that $\delta$ is small compared to the constant $\varepsilon_{2}$ in Lemma 4.3.

We may replace $\mathcal{S}_{t}^{\delta}$ by

$$
T_{t}^{\delta}=\psi(D) \mathcal{S}_{t}^{\delta}
$$


where $\psi$ is as in Lemma 4.3 (smooth and supported in a ball of radius $\varepsilon_{1}$ ). In view of the invariance properties of the weight operators one can use a partition of unity to deduce the weighted inequality (37) from the corresponding result for $T_{t}^{\delta}$.

We now prepare for an application of Lemma 4.3 and decompose on the frequency side the product $F_{1} \overline{F_{2}}$ for suitable $F_{i}$ (initially $F_{i}=T_{t}^{\delta} f_{i}$ ). We let $\chi_{\circ}$ to be a radial $C_{c}^{\infty}$ function $\chi_{\circ}(\omega)=1$ for $|\omega| \leq 2^{5}$ and so that $\operatorname{supp} \chi_{\circ}$ is contained in $\left\{\omega:|\omega| \leq 2^{5}+1\right\}$, moreover set,

$$
\chi_{1}(\omega)=\chi_{\circ}(\omega)-\chi_{\circ}(2 \omega) .
$$

We also let $j_{\circ}=j_{\circ}(\delta)$ denote the integer with

$$
\sqrt{\delta / \varepsilon_{2}} \leq 2^{-j_{\circ}}<2 \sqrt{\delta / \varepsilon_{2}}
$$

where $\varepsilon_{2} \in(0,1)$ is as in Lemma 4.3 .

Define bilinear forms for pairs of Schwartz functions by

$$
\begin{aligned}
\mathfrak{B}_{\circ}\left[F_{1}, F_{2}\right](x) & =\frac{1}{(2 \pi)^{2 d}} \iint \chi_{\circ}\left(2^{j \circ}(\xi-\eta)\right) \widehat{F}_{1}(\xi) \widehat{F}_{2}(-\eta) e^{\imath\langle\xi-\eta, x\rangle} d \xi d \eta, \\
\mathcal{B}^{j}\left[F_{1}, F_{2}\right](x) & =\frac{1}{(2 \pi)^{2 d}} \iint \chi_{1}\left(2^{j}(\xi-\eta)\right) \widehat{F}_{1}(\xi) \widehat{F}_{2}(-\eta) e^{\imath\langle\xi-\eta, x\rangle} d \xi d \eta .
\end{aligned}
$$

Then one easily verifies the decomposition

$$
F_{1} \overline{F_{2}}=\mathfrak{B}_{\circ}\left[F_{1}, \overline{F_{2}}\right]+\sum_{j<j} \mathcal{B}^{j}\left[F_{1}, \overline{F_{2}}\right] .
$$

Later, in cases where the supports of the Fourier transforms of $F_{1}$ and $F_{2}$ are separated we wish to dispense with the frequency cutoff $\Phi_{j}(\xi-\eta)$ and replace $\mathfrak{B}_{\circ}$ or $\mathcal{B}_{j}$ by a product. This will be accomplished by using the identities

$$
\begin{aligned}
(2 \pi)^{d} \mathfrak{B}_{\circ}\left[F_{1}, F_{2}\right](x) & =2^{-j_{0} d} \widehat{\chi}_{\circ}\left(2^{-j_{0}} \cdot\right) *\left[F_{1} F_{2}\right](x), \\
(2 \pi)^{d} \mathcal{B}^{j}\left[F_{1}, F_{2}\right](x) & =2^{-j d} \widehat{\chi}_{1}\left(2^{-j} \cdot\right) *\left[F_{1} F_{2}\right](x)
\end{aligned}
$$

which follow from the Fourier inversion formula (and the assumption that $\chi_{\circ}$ is radial).

The desired weighted norm inequality (37) follows from the following two propositions, applied for $f_{1}=f_{2}=f$. The proof of the first one is rather straightforward.

\section{Proposition 4.5.}

$$
\left|\iint_{1}^{2} \mathfrak{B}_{\circ}\left[T_{t}^{\delta} f_{1}, \overline{T_{t}^{\delta} f_{2}}\right](x) \frac{d t}{t} w(x) d x\right| \lesssim \delta \prod_{i=1,2}\left(\int\left|f_{i}(x)\right|^{2} \int \frac{\delta^{d / 2} \mathfrak{M}_{\sqrt{\delta}} w(x-y)}{\left(1+\delta^{1 / 2}|y|\right)^{d+1}} d y d x\right)^{1 / 2} .
$$

More substantial (and relying on $₫ 3$ ) is

Proposition 4.6. Let $q \in\left[1, \frac{d+2}{2}\right)$. Then, for $j<j_{\circ}$,

$$
\begin{aligned}
\mid \iint_{1}^{2} \mathcal{B}^{j}\left[T_{t}^{\delta} f_{1}, \overline{T_{t}^{\delta} f_{2}}\right](x) & \frac{d t}{t} w(x) d x \mid \\
& \lesssim \delta^{2-\frac{d}{q}} 2^{-2 j\left(\frac{d}{q}-1\right)} \prod_{i=1,2}\left(\max _{|\nu| \leq 8} \int\left|f_{i}(x)\right|^{2} H_{j} * \mathcal{W}_{q, \delta, 0}^{j-\nu} w(x) d x\right)^{1 / 2}
\end{aligned}
$$

where $H_{j}(x)=2^{-j d}\left(1+2^{-j}|x|\right)^{-d-1}$. 
We now introduce some basic decompositions. As in the definition (38) let $\zeta \in C_{c}^{\infty}\left(\mathbb{R}^{d}\right)$ be supported in $\{y:|y| \leq 1 / 8\}$, now with the additional assumption that $\zeta(y)=1$ for $|y| \leq 1 / 9$. Let $\widetilde{\zeta} \in C_{c}^{\infty}\left(\mathbb{R}^{d}\right)$ be supported in $\{y:|y| \leq 1 / 7\}$ so that $\widetilde{\zeta}(y)=1$ for $|y| \leq 1 / 8$; hence $\widetilde{\zeta} \zeta=\zeta$. Let $\varphi$ be a smooth function supported in $[-9 / 8,9 / 8]$ and equal to 1 on $[-7 / 8,7 / 8]$ such that

$$
\sum_{n \in \mathbb{Z}} \varphi(\cdot-n)=1
$$

We let $\Theta_{j}$ be a maximal $2^{-j-d}$-separated set of $S^{d-1}$ and define for $n \in \mathbb{Z}, l \in \mathbb{Z}$, operators via the Fourier transform by

$$
\begin{aligned}
\mathcal{F}\left[Q_{n}^{\theta, j} f\right](\xi) & =\frac{\zeta\left(2^{j}\left(\frac{\xi}{|\xi|}-\theta\right)\right)}{\sum_{\theta^{\prime} \in \Theta_{j}} \zeta^{2}\left(2^{j}\left(\frac{\xi}{|\xi|}-\theta^{\prime}\right)\right)} \varphi\left(2^{j}|\xi|-n\right) \widehat{f}(\xi), \\
\mathcal{F}\left[P_{l}^{\theta, j} f\right](\xi) & =\widetilde{\zeta}\left(2^{j}\left(\frac{\xi}{|\xi|}-\theta\right)\right) \varphi\left(2^{2 j}|\xi|-l\right) \widehat{f}(\xi)
\end{aligned}
$$

moreover, with $Q^{\theta, j}$ as in (38) set

$$
\mathcal{Q}^{\theta, j}=\sum_{n} Q^{\theta, j} Q_{n}^{\theta, j}
$$

so that

$$
T_{t}^{\delta} f=\sum_{\theta \in \Theta_{j}} T_{t}^{\delta} \mathcal{Q}^{\theta, j} f=\sum_{\theta \in \Theta_{j}} \sum_{n \in \mathbb{Z}} \sum_{l \in \mathbb{Z}} T_{t}^{\delta} Q^{\theta, j} Q_{n}^{\theta, j} P_{l}^{\theta, j} f,
$$

for both cases $j<j_{\circ}$ and $j=j_{\circ}$.

Note that the multipliers for $Q^{\theta, j}$ and $\mathcal{Q}^{\theta, j}$ are contained in a sector of width $c 2^{-j}$ around $\theta$. The multiplier for $Q_{n}^{\theta, j}$ is contained in a $c 2^{-j}$ ball centered around $\Xi$ with $|\Xi|=2^{-j} n+O\left(2^{-j}\right)$. The multiplier for $P_{l}^{\theta, j}$ is contained in a plate with $(d-1)$ sides of length $O\left(2^{-j}\right)$ and a short side of length $O\left(2^{-2 j}\right)$, the long sides being perpendicular to $\theta$. Note that for $2^{-2 j} m \in[1,2]$

$$
\begin{gathered}
T_{t}^{\delta} Q^{\theta, j} Q_{n}^{\theta, j} P_{l}^{\theta, j} \neq 0 \text { for some } t \in\left[(m-1) 2^{-2 j},(m+1) 2^{-2 j}\right], \\
\Longrightarrow|m-l| \lesssim 1, \quad\left|2^{-2 j} l-2^{-j} n\right| \lesssim 2^{-j} .
\end{gathered}
$$

We also notice that, from the localization and separation properties of the cutoff functions $\chi_{\circ}\left(2^{j_{\circ}}(\xi-\eta)\right)$ and $\chi_{1}\left(2^{j}(\xi-\eta)\right)$ in (41),

$$
\begin{aligned}
\mathfrak{B}_{\circ}\left(T_{t}^{\delta} Q^{\theta, j} g, \overline{T_{t}^{\delta} Q^{\theta^{\prime}, j} g}\right) \neq 0 & \Longrightarrow \operatorname{dist}\left(\theta, \theta^{\prime}\right) \leq C 2^{-j \circ}, \\
\mathcal{B}_{j}\left(T_{t}^{\delta} Q^{\theta, j} g, \overline{T_{t}^{\delta} Q^{\theta^{\prime}, j} g}\right) \neq 0 & \Longrightarrow 2^{-j+3} \leq \operatorname{dist}\left(\theta, \theta^{\prime}\right) \leq 2^{-j+6} .
\end{aligned}
$$

Proof of Proposition 4.5. We first observe by a straightforward integration by parts that for $t \in[1,2]$ the convolution kernel associated with $T_{t}^{\delta} Q^{\theta, j_{\circ}}$ is dominated by

$$
C_{d} \frac{\delta^{\frac{d+1}{2}}}{\left(1+\delta|\langle x, \theta\rangle|+\delta^{1 / 2}|x-\langle x, \theta\rangle \theta|\right)^{N}} \lesssim \mathcal{K}_{2^{-j_{\circ}}}^{\theta, j_{\circ}}(x) \approx \mathcal{K}_{2^{j \circ \delta}}^{\theta, j_{\circ}}(x) .
$$

On the right hand side we may also replace $\theta$ by any $\widetilde{\theta}$ with $|\theta-\widetilde{\theta}| \lesssim 2^{-j \circ}$. For more compact notation we write

$$
\mathcal{K}^{\theta, j_{\circ}}(x):=\mathcal{K}_{2^{-j_{\circ}}}^{\theta, j_{\circ}}(x)
$$


Now for $\left|\theta_{1}-\theta_{2}\right| \lesssim 2^{-j \circ}$ and each $t \in[1,2]$, we have

$$
\begin{aligned}
& \left|\int \prod_{i=1,2}^{*}\left[T_{t}^{\delta} Q^{\theta_{i}, j_{\circ}} g_{i}(x)\right] w(x) d x\right| \lesssim \int\left[\prod_{i=1,2}\left(\mathcal{K}^{\theta_{i}, j_{\circ}} *\left|g_{i}\right|^{2}(x)\right)^{1 / 2}\right]|w(x)| d x \\
& \lesssim \prod_{i=1,2}\left(\int \mathcal{K}^{\theta_{i}, j_{\circ}} *\left|g_{i}\right|^{2}(x)|w(x)| d x\right)^{1 / 2} \lesssim \prod_{i=1,2}\left(\int\left|g_{i}(y)\right|^{2} \mathcal{K}^{\theta_{i}, j_{\circ}} *|w|(y) d y\right)^{1 / 2}
\end{aligned}
$$

here we used $\left\|\mathcal{K}^{\theta, j_{\circ}}\right\|_{1}=O(1)$ and applied the Schwarz inequality twice.

By Lemma 4.4

$$
\sum_{l}\left|P_{l}^{\theta, j_{\circ}} f(x)\right|^{2} \lesssim \mathcal{K}^{\theta_{i}, j_{\circ}} *|f|^{2}(x)
$$

Now let $I_{m}^{j_{\circ}}=\left[2^{-2 j_{\circ}} m, 2^{-2 j_{\circ}}(m+1)\right]$. It will be implicit in all $m$-summations that $I_{m}^{j_{\circ}} \subset[1,2]$. In what follows $\mathfrak{A}_{j_{\circ}}^{m}$ will be an index set consisting of $\left(l_{1}, l_{2}, n_{1}, n_{2}\right)$ with $\left|l_{i}-m\right| \lesssim 1$, $\left|2^{-2 j_{\circ}} l_{i}-2^{-j_{\circ}} n_{i}\right| \lesssim 2^{-j_{\circ}}$ for $i=1,2$. Then

$$
\begin{aligned}
& \left|\sum_{\substack{\theta_{1}, \theta_{2} \in \Theta_{j} \\
\left|\theta_{1}-\theta_{2}\right| \leq C 2^{-j \circ}}} \iint_{1}^{2} \prod_{i=1,2}^{*}\left[T_{t}^{\delta} \mathcal{Q}^{\theta_{i}, j_{\circ}} f_{i}(x)\right] \frac{d t}{t} w(x) d x\right| \\
= & \left|\sum_{\substack{\theta_{1}, \theta_{2} \in \Theta_{j} \\
\left|\theta_{1}-\theta_{2}\right| \leq C 2^{-j \circ}}} \sum_{m} \int_{I_{m}^{j}} \sum_{\substack{\left(l_{1}, l_{2}, n_{1}, n_{2}\right) \\
\in \mathfrak{A}_{j \circ}^{m}}} \int \prod_{i=1,2}^{*}\left[T_{t}^{\delta} Q^{\theta_{i}, j_{\circ}} P_{l_{i}}^{\theta_{i}, j_{\circ}} Q_{n_{i}}^{\theta_{i}, j_{\circ}} f_{i}(x)\right] w(x) d x \frac{d t}{t}\right| \\
\lesssim & 2^{-2 j_{\circ}} \sum_{\substack{\theta_{1}, \theta_{2} \in \Theta_{j \circ} \\
\left|\theta_{1}-\theta_{2}\right| \leq C 2^{-j \circ}}} \sum_{m} \sum_{\substack{\left(l_{1}, l_{2}, n_{1}, n_{2}\right) \\
\in \mathfrak{A}_{j \circ}^{m}}} \prod_{i=1,2}\left(\int\left|P_{l_{i}}^{\theta_{i}, j_{\circ}} Q_{n_{i}}^{\theta_{i}, j_{\circ}} f_{i}(x)\right|^{2} \mathcal{K}^{\theta_{i}, j_{\circ}} *|w|(x) d y\right)^{1 / 2} ;
\end{aligned}
$$

here we have applied (45) and carried out the $t$ integration. We now notice that $\left|n_{1}-n_{2}\right| \lesssim 1$, $\left|l_{1}-l_{2}\right| \lesssim 1$ for $\left(l_{1}, l_{2}, n_{1}, n_{2}\right) \in \mathfrak{A}_{j_{\circ}}^{m}$ and that for fixed $\left(l_{1}, l_{2}, n_{1}, n_{2}\right)$ there are only $O(1)$ integers $m$ for which $\left(l_{1}, l_{2}, n_{1}, n_{2}\right) \in \mathfrak{A}_{j_{\circ}}^{m}$. Hence, by various applications of the Schwarz inequality and then by (46) the last displayed quantity is controlled by

$$
\begin{aligned}
& 2^{-2 j \circ} \prod_{i=1,2}\left(\sum_{\theta_{i} \in \Theta_{j}} \sum_{l_{i}} \sum_{n_{i}} \int\left|P_{l_{i}}^{\theta_{i}, j_{\circ}} Q_{n_{i}}^{\theta_{i}, j_{\circ}} f_{i}(x)\right|^{2} \mathcal{K}^{\theta_{i}, j_{\circ}} *|w|(x) d x\right)^{1 / 2} \\
\lesssim & 2^{-2 j \circ} \prod_{i=1,2}\left(\sum_{\theta_{i} \in \Theta_{j_{\circ}}} \sum_{n_{i}} \int\left|Q_{n_{i}}^{\theta_{i}, j_{\circ}} f_{i}(x)\right|^{2} \mathcal{K}^{\theta_{i}, j_{\circ}} * \mathcal{K}^{\theta_{i}, j_{\circ}} *|w|(x) d y\right)^{1 / 2}
\end{aligned}
$$

where, by Lemma 2.3, we may replace $\mathcal{K}^{\theta_{i}, j_{\circ}} * \mathcal{K}^{\theta_{i}, j_{\circ}}$ with $\mathcal{K}^{\theta_{i}, j_{\circ}}$.

By Lemma 4.4 we have, with $H_{j_{\circ}}(x)=2^{-j_{\circ} d}\left(1+2^{-j_{\circ}}|x|\right)^{-d-1}$,

$$
\sum_{\theta \in \Theta_{j_{\circ}}} \sum_{n}\left|Q_{n}^{\theta, j_{\circ}} f\right|^{2} \lesssim H_{j_{\circ}} *|f|^{2}(x)
$$

so that we may estimate the term (47) by

$$
2^{-2 j \circ} \prod_{i=1,2}\left(\int\left|f_{i}(x)\right|^{2} H_{j_{\circ}} * \sup _{\theta}\left[\mathcal{K}^{\theta, j_{\circ}} *|w|\right](x) d x\right)^{1 / 2} .
$$


Now by the definition of $\mathfrak{B}_{\circ}$, (42) and translation invariance

$$
\begin{aligned}
\mathfrak{B}_{\circ}\left[T_{t}^{\delta} f_{1}, \overline{T_{t}^{\delta} f_{2}}\right](x)= & \sum_{\substack{\theta_{1}, \theta_{2} \in \Theta_{j_{\circ}} \\
\left|\theta_{1}-\theta_{2}\right| \leq C 2^{-j_{\circ}}}} \mathfrak{B}_{\circ}\left[T_{t}^{\delta} \mathcal{Q}^{\theta_{1}, j_{\circ}} f_{1}, \overline{T_{t}^{\delta} \mathcal{Q}^{\theta_{2}, j_{\circ}} f_{2}}\right](x) \\
= & \sum_{\substack{\theta_{1}, \theta_{2} \in \Theta_{j_{\circ}} \\
\left|\theta_{1}-\theta_{2}\right| \leq C 2^{-j_{\circ}}}} \frac{1}{(2 \pi)^{d}} \int 2^{-j_{\circ}} d \widehat{\chi}_{\circ}\left(2^{-j_{\circ}} h\right) \prod_{i=1,2}^{*}\left[T_{t}^{\delta} \mathcal{Q}^{\theta_{i}, j_{\circ}} \tau_{h} f_{i}\right](x) d h
\end{aligned}
$$

where $\tau_{h} f(x)=f(x-h)$. We combine this identity with the previous estimate and the obvious inequality $\left|2^{-j_{\circ} d} \widehat{\chi}_{\circ}\left(2^{-j_{\circ}} \cdot\right)\right| \lesssim H_{j_{\circ}}$ to obtain

$$
\begin{aligned}
& \left|\iint_{1}^{2} \mathfrak{B}_{\circ}\left[T_{t}^{\delta} f_{1}, \overline{T_{t}^{\delta} f_{2}}\right](x) \frac{d t}{t} w(x) d x\right| \\
\lesssim & \int H_{j_{\circ}}(h)\left|\iint_{1}^{2} \sum_{\substack{\theta_{1}, \theta_{2} \in \Theta_{\Theta_{\circ}} \\
\left|\theta_{1}-\theta_{2}\right| \leq C 2^{-j_{\circ}}}} \prod_{i=1,2}^{*}\left[T_{t}^{\delta} \mathcal{Q}^{\theta_{i}, j_{\circ}} \tau_{h} f_{i}\right](x) \frac{d t}{t} w(x) d x\right| d h \\
\lesssim & \int H_{j_{\circ}}(h) 2^{-2 j_{\circ}} \prod_{i=1,2}\left(\int\left|f_{i}(x-h)\right|^{2} H_{j_{\circ}} * \sup _{\theta}\left[\mathcal{K}^{\theta, j_{\circ}} *|w|\right](x) d x\right)^{1 / 2} d h .
\end{aligned}
$$

Clearly $\sup _{\theta}\left[\mathcal{K}^{\theta, j_{\circ}} *|w|\right] \lesssim \mathfrak{M}_{\sqrt{\delta}}[w]$. By the Schwarz inequality and a subsequent change of variable the last displayed quantity is bounded by

$$
C \delta \prod_{i=1,2}\left(\sum_{\theta_{i} \in \Theta_{j \circ}} \int\left|f_{i}(x)\right|^{2} H_{j_{\circ}} * H_{j_{\circ}} * \mathfrak{M}_{\sqrt{\delta}}[w](x) d x\right)^{1 / 2} .
$$

Since $H_{j_{\circ}} * H_{j_{\circ}}(x) \lesssim \delta^{d / 2}\left(1+\delta^{1 / 2}|x|\right)^{-d-1}$, by Lemma 2.3, this concludes the proof of the proposition.

Proof of Proposition 4.6. We fix $j<j$ 。 so that $2^{2 j} \delta<1 / 2$. We define that $\theta \sim \theta^{\prime}$ if $\theta \in \Theta_{j}, \theta^{\prime} \in \Theta_{j}$ and $2^{-j+3} \leq\left|\theta-\theta^{\prime}\right| \leq 2^{-j+6}$; this is the relevant range in (44).

Below we shall prove the estimate

$$
\begin{aligned}
\mid \iint_{1}^{2} \prod_{i=1,2}^{*}\left[T_{t}^{\delta}\right. & \left.\mathcal{Q}^{\theta_{i}, j} f_{i}(x)\right] \frac{d t}{t} w(x) d x \mid \\
& \quad \delta^{2-\frac{d}{q}} 2^{-2 j\left(\frac{d}{q}-1\right)} \prod_{i=1,2}\left(\sum_{n} \int\left|Q_{n}^{\theta_{i}, j} f_{i}(x)\right|^{2}\left(\mathcal{K}_{2^{j} \delta}^{\theta_{i}, j} *|w|^{q}(x)\right)^{1 / q} d x\right)^{1 / 2}
\end{aligned}
$$

for any $\theta_{1}, \theta_{2}$ with $\theta_{1} \sim \theta_{2}$. We first show why (49) implies the asserted estimate.

It is crucial to observe that for $\theta_{1} \sim \theta_{2}$ and any $g_{1}, g_{2}$ the Fourier transform of the product $T_{t}^{\delta} Q^{\theta_{1}, j} g_{1} \overline{T_{t}^{\delta} Q^{\theta_{2}, j} g_{2}}$ is supported in

$$
\left\{\xi: 2^{-j+2} \leq|\xi| \leq 2^{-j+6},|\langle\xi, \vartheta\rangle| \leq 2^{-2 j+12}\right\} ;
$$

here $\vartheta=\frac{\theta_{1}+\theta_{2}}{\left|\theta_{1}+\theta_{2}\right|}$. Let $\eta_{0} \in C_{c}^{\infty}(\mathbb{R})$ be even and supported in $\left[-2^{13}, 2^{13}\right]$ so that $\eta_{0}(s)=1$ for $|s| \leq 2^{12}$ and let $\eta_{1} \in C_{c}^{\infty}(\mathbb{R})$ be supported in $[2,128]$ so that $\eta_{1}(s)=1$ for $s \in[4,64]$. Consider the even Schwartz function $\Psi \equiv \Psi^{j, \theta_{1}, \theta_{2}}$ defined by

$$
\widehat{\Psi^{j, \theta_{1}, \theta_{2}}}(\xi)=\eta_{0}\left(2^{2 j}\langle\xi, \vartheta\rangle\right) \eta_{1}\left(2^{j}|\xi|\right) \text {. }
$$


Note that there is a constant $C$ (independent of $j, \theta_{1}, \theta_{2}$ ) so that

$$
C^{-1} \Psi^{j, \theta_{1}, \theta_{2}} \in \mathcal{S}_{j-\nu}^{\theta_{i}, j}
$$

for $\nu=0, \ldots, 8, i=1,2$.

From these considerations it follows that

$$
\int \prod_{i=1,2}^{*}\left[T_{t}^{\delta} \mathcal{Q}^{\theta_{i}, j} f_{i}\right](x) w(x) d x=\sum_{|\nu| \leq 8} \int \prod_{i=1,2}^{*}\left[T_{t}^{\delta} \mathcal{Q}^{\theta_{i}, j} f_{i}\right](x) \Psi^{j, \theta_{1}, \theta_{2}} * P_{\nu-j} w(x) d x
$$

and therefore, for any $\theta_{1}, \theta_{2}$ with $\theta_{1} \sim \theta_{2}$, (49) can be changed to

$$
\left|\iint_{1}^{2} \prod_{i=1,2}^{*}\left[T_{t}^{\delta} \mathcal{Q}^{\theta_{i}, j}\right](x) \frac{d t}{t} w(x) d x\right| \lesssim \prod_{i=1,2}\left(\sum_{n} \int\left|Q_{n}^{\theta_{i}, j} f_{i}(x)\right|^{2} U_{q, \delta}^{\theta_{i}, j} w(x) d x\right)^{1 / 2},
$$

where

$$
U_{q, \delta}^{\theta, j} w(x)=\delta^{2-\frac{d}{q}} 2^{-2 j\left(\frac{d}{q}-1\right)} \sum_{|\nu| \leq 8}\left(\mathcal{K}_{2^{j} \delta}^{\theta, j} * \sup _{\Psi \in \mathcal{S}_{\nu-j}^{\theta, j}}\left|\Psi * P_{\nu-j} w\right|^{q}(x)\right)^{1 / q} .
$$

By the definition of $\mathcal{B}_{j}$ we have

$$
\begin{aligned}
\mathcal{B}^{j}\left[T_{t}^{\delta} f_{1}, \overline{T_{t}^{\delta} f_{2}}\right](x) & =\sum_{\theta_{1} \sim \theta_{2}} \mathcal{B}^{j}\left[T_{t}^{\delta} \mathcal{Q}^{\theta_{1}, j} f_{1}, \overline{T_{t}^{\delta} \mathcal{Q}^{\theta_{2}, j} f_{2}}\right](x) \\
& =\sum_{\theta_{1} \sim \theta_{2}}(2 \pi)^{-d} \int_{h} 2^{-j d} \widehat{\chi}_{1}\left(2^{-j} h\right) \prod_{i=1,2}^{*}\left[T_{t}^{\delta} \mathcal{Q}^{\theta_{i}, j} \tau_{h} f_{i}\right](x) d h .
\end{aligned}
$$

Therefore (50) yields

$$
\begin{aligned}
& \left|\iint_{1}^{2} \mathcal{B}^{j}\left[T_{t}^{\delta} f_{1}, \overline{T_{t}^{\delta} f_{2}}\right](x) \frac{d t}{t} w(x) d x\right| \\
\lesssim & \int H_{j}(h) \prod_{i=1,2}\left(\sum_{\theta_{i} \in \Theta_{j}} \sum_{n} \int\left|Q_{n}^{\theta_{i}, j} f_{i}(x-h)\right|^{2} U_{q, \delta}^{\theta_{i}, j} w(x) d x\right)^{1 / 2} \\
\lesssim & \prod_{i=1,2}\left(\sum_{\theta_{i} \in \Theta_{j}} \sum_{n} \int\left|Q_{n}^{\theta_{i}, j} f_{i}(x)\right|^{2} H_{j} *\left[\sup _{\theta} U_{q, \delta}^{\theta, j} w\right](x) d x\right)^{1 / 2},
\end{aligned}
$$

by the Schwarz inequality. Now, for $|\nu| \leq 8$, observe $\mathcal{K}_{2^{j} \delta}^{\theta, j} \approx \mathcal{K}_{2^{j-\nu} \delta}^{\theta, j-\nu}$ and therefore

$$
\sup _{\theta} U_{q, \delta}^{\theta, j} w(x) \lesssim \delta^{2-\frac{d}{q}} 2^{-2 j\left(\frac{d}{q}-1\right)} \mathcal{W}_{q, \delta, 0}^{j-\nu} w(x) .
$$

Moreover, by Lemma 4.4,

$$
\sum_{\theta \in \Theta_{j}} \sum_{n}\left|Q_{n}^{\theta, j} f(x)\right|^{2} \lesssim H_{j} *|f|^{2}(x) .
$$

Hence, using the Schwarz inequality again, we get

$$
\begin{aligned}
\mid \iint_{1}^{2} \mathcal{B}^{j}\left[T_{t}^{\delta} f_{1}, \overline{T_{t}^{\delta} f_{2}}\right] & (x) \frac{d t}{t} w(x) d x \mid \\
& \lesssim \delta^{2-\frac{d}{q}} 2^{-2 j\left(\frac{d}{q}-1\right)} \max _{|\nu| \leq 8} \prod_{i=1,2}\left(\int\left|f_{i}(x)\right|^{2} H_{j} * H_{j} * \mathcal{W}_{q, \delta, 0}^{j-\nu} w(x) d x\right)^{1 / 2}
\end{aligned}
$$

and since $H_{j} * H_{j} \lesssim H_{j}$ we obtain the asserted estimate. 
Proof of (49). We argue as in the proof of Proposition 4.5 and rely on Lemma 4.3. We let $I_{m}^{j}=\left[2^{-2 j} m, 2^{-2 j}(m+1)\right]$ if this interval is a subset of $[1,2]$ (otherwise $I_{m}^{j}=\emptyset$ ). Define the index sets $\mathfrak{A}_{j}^{m}$ as in the proof of Proposition 4.5 (with $j$ instead of $j_{\circ}$ ).

Then the right hand side of (49) is equal to

$$
\left|\sum_{m} \sum_{\left(l_{1}, l_{2}, n_{1}, n_{2}\right) \in \mathfrak{A}_{j}^{m}} \iint_{I_{m}^{j}} \prod_{i=1,2}^{*}\left[T_{t}^{\delta} Q^{\theta_{i}, j} Q_{n_{i}}^{\theta_{i}, j} P_{l_{i}}^{\theta_{i}, j} f_{i}(x)\right] \frac{d t}{t} w(x) d x\right|
$$

and, by Lemma 4.3, this is estimated by

$$
\begin{aligned}
& \sum_{m} \sum_{\substack{\left|l_{1}-m\right| \lesssim 1 \\
\left|l_{2}-m\right| \lesssim 1}} \sum_{\left|n_{1}-n_{2}\right| \lesssim 1} \delta^{2-\frac{d}{q}} 2^{-2 j\left(\frac{d}{q}-1\right)} \prod_{i=1,2}\left(\int\left|P_{l_{i}}^{\theta_{i}, j} Q_{n_{i}}^{\theta_{i}, j} f_{i}(x)\right|^{2}\left(\mathcal{K}_{2^{j} \delta}^{\theta_{i}, j} *|w|^{q}(x)\right)^{1 / q} d x\right)^{1 / 2} \\
& \lesssim \delta^{2-\frac{d}{q}} 2^{-2 j\left(\frac{d}{q}-1\right)} \prod_{i=1,2}\left(\sum_{n_{i}} \sum_{l_{i}} \int\left|P_{l_{i}}^{\theta_{i}, j} Q_{n_{i}}^{\theta_{i}, j} f_{i}(x)\right|^{2}\left(\mathcal{K}_{2^{j} \delta}^{\theta_{i}, j} *|w|^{q}(x)\right)^{1 / q} d x\right)^{1 / 2} \\
& \lesssim \delta^{2-\frac{d}{q}} 2^{-2 j\left(\frac{d}{q}-1\right)} \prod_{i=1,2}\left(\sum_{n_{i}} \int\left|Q_{n}^{\theta_{i}, j} f_{i}(x)\right|^{2} \mathcal{K}_{2^{-j}}^{\theta_{i}, j} *\left(\mathcal{K}_{2^{j} \delta}^{\theta_{i}, j} *|w|^{q}(x)\right)^{1 / q} d x\right)^{1 / 2} ;
\end{aligned}
$$

here we have used the Schwarz inequality and the bound

$$
\sum_{l}\left|P_{l}^{\theta, j} g(x)\right|^{2} \lesssim \mathcal{K}_{2^{-j}}^{\theta, j} *|g|^{2}(x)
$$

which is a consequence of Lemma 4.4. We also have

$$
\mathcal{K}_{2^{-j}}^{\theta, j} *\left(\mathcal{K}_{2^{j} \delta}^{\theta, j} *|w|^{q}(x)\right)^{1 / q} \lesssim\left(\mathcal{K}_{2^{-j}}^{\theta, j} * \mathcal{K}_{2^{j} \delta}^{\theta, j} *|w|^{q}(x)\right)^{1 / q} \lesssim\left(\mathcal{K}_{2^{j} \delta}^{\theta, j} *|w|^{q}(x)\right)^{1 / q} ;
$$

where the first estimate follows from Hölder's inequality and the second from (18) and the assumption $2^{j} \delta<2^{-j}$. This completes the proof of (49) and thus the proposition is established.

\section{5. $L^{p}\left(L^{2}\right)$ EStimates For SOlutions of Schrödinger AND WAVE EQUATions}

Proposition 5.1. Let $d \geq 2$ and $p \in\left(\frac{2(d+2)}{d}, \infty\right]$, or $d=1$ and $p=\infty$, and let $a \in(0, \infty)$. Let $I$ denote a compact interval of time. Then for $k \geq 1$,

$$
\left\|\left(\int_{I}\left|U_{t}^{a} P_{k} f\right|^{2} d t\right)^{1 / 2}\right\|_{p} \lesssim 2^{k a \lambda(p)}\|f\|_{L^{p}}, \quad \lambda(p)=d\left(\frac{1}{2}-\frac{1}{p}\right)-\frac{1}{2} .
$$

Proof of Theorem 1.4. The result is an immediate consequence of Proposition 5.1 and the case $q=2, r=1, v=p$ of Proposition A.3.

Proof of Proposition 5.1. We may assume that $2^{k}$ is large. Let $\phi$ be an even real-valued function so that $\widehat{\phi}(t)>c>0$ for $t \in I$ and $\operatorname{supp} \phi \subset[-1 / 4,1 / 4]$. It suffices to estimate the $L^{p}\left(\mathbb{R}^{d}, L^{2}(\mathbb{R})\right)$ norm of $\widehat{\phi}(t) U_{t}^{a} P_{k} f(x)$. For fixed $x$ the $L^{2}(\mathbb{R})$ norm of this expression is equal to

$$
\begin{aligned}
& \frac{1}{(2 \pi)^{1 / 2}}\left(\int_{\mathbb{R}}\left|\int \widehat{\phi}(t) \exp (-\imath t \tau) U_{t}^{a} P_{k} f(x) d t\right|^{2} d \tau\right)^{1 / 2} \\
= & \frac{1}{(2 \pi)^{1 / 2}}\left(\int_{2^{(k-3) a}}^{2^{(k+3) a}}\left|\mathcal{F}^{-1}\left[\phi\left(|\cdot|^{a}-\tau\right) \chi\left(2^{-k}|\cdot|\right) \widehat{f}\right](x)\right|^{2} d \tau\right)^{1 / 2} .
\end{aligned}
$$


By a finite splitting we may replace the integral over $\left[2^{(k-3) a}, 2^{(k+3) a}\right]$ by an integral over $\left(\left(2^{k} T\right)^{a},\left(2^{k+1} T\right)^{a}\right)$ with $T \approx 1$. After changing variables $\tau=\left(2^{k} T r\right)^{a}$ it suffices to show that

$$
\left\|\left(\int_{1}^{2}\left|\mathcal{F}^{-1}\left[\phi\left(|\cdot|^{a}-\left(2^{k} T r\right)^{a}\right) \chi\left(2^{-k}|\cdot|\right) \widehat{f}\right]\right|^{2} d r\right)^{1 / 2}\right\|_{p} \lesssim 2^{a\left(\lambda(p)-\frac{1}{2}\right) k}\|f\|_{p},
$$

or, after scaling and setting $\delta=\left(2^{k} T\right)^{-a}$,

$$
\left\|\left(\int_{1}^{2}\left|\mathcal{F}^{-1}\left[\phi\left(\delta^{-1}\left(|\cdot|^{a}-r^{a}\right)\right) \chi(T|\cdot|) \widehat{f}\right]\right|^{2} d r\right)^{1 / 2}\right\|_{p} \lesssim \delta^{\frac{1}{2}-\lambda(p)}\|f\|_{p} .
$$

But as $\phi$ is even, $\phi\left(\delta^{-1}\left(|\xi|^{a}-r^{a}\right)\right)=\phi\left(\delta^{-1} \beta(\xi, r)\left(1-|\xi|^{2} / r^{2}\right)\right)$ where $\beta(\xi, r)=r^{2} \frac{r^{a}-|\xi|^{a}}{r^{2}-|\xi|^{2}}$ is smooth for $\xi$ away from the origin, and nonvanishing. Thus Corollary 4.2 may be applied and we get the $L^{p}$ inequality (52) for $d \geq 2$ and $p>2+4 / d$.

The case $d=1, p=\infty$ is more straightforward; the estimate

$$
\left(\int_{1}^{2}\left|\mathcal{F}^{-1}\left[\phi\left(\delta^{-1}\left(|\cdot|^{a}-r^{a}\right)\right) \chi_{1}(T|\cdot|) \widehat{f}\right](x)\right|^{2} d r\right)^{1 / 2} \lesssim \delta^{1 / 2}\|f\|_{\infty}
$$

for $T \approx 1$ follows from

$$
\sum_{0 \leq n<\delta^{-1}} \mid \mathcal{F}^{-1}\left[\left.\phi\left(\delta^{-1}\left(|\cdot|^{a}-(1+n \delta+\sigma)^{a}\right) \chi_{1}(T|\cdot|) \widehat{f}\right](x)\right|^{2} \lesssim\|f\|_{\infty}^{2}, \quad 0<\sigma \leq \delta,\right.
$$

and integration in $\sigma$. The last displayed inequality however is a consequence of Lemma 4.4.

We finish by stating a global variant of the one-dimensional square function estimate which does not use Sobolev spaces and which we will not use elsewhere in the paper.

Proposition 5.2. Let $d=1, p \in[2, \infty), a \in(0, \infty)$, and let $I$ be a compact interval. Then

$$
\left\|\left(\int_{I}\left|U_{t}^{a} f\right|^{2} d t\right)^{1 / 2}\right\|_{L^{p}(\mathbb{R})} \lesssim\|f\|_{L^{p}(\mathbb{R})}
$$

Moreover

$$
\left\|\left(\int_{I}\left|U_{t}^{a} f\right|^{2} d t\right)^{1 / 2}\right\|_{B M O(\mathbb{R})} \lesssim\|f\|_{L^{\infty}(\mathbb{R})}
$$

Given the reduction in the localized case, in the proof of Proposition 5.1, the $L^{p}\left(L^{2}\right)$ estimates can be deduced from a regularized version of Rubio de Francia's square function estimate [31] associated with arbitrary disjoint collection of intervals. The $L^{\infty}-B M O$ estimate can be obtained from Sjölin's proof [35] of that estimate. We omit the details.

\section{An $L^{p}\left(L^{q}\right)$ estimate}

We state the $L^{p}\left(L^{q}\right)$ estimates alluded to in the introduction. We work with the norm

$$
\|u\|_{L^{p}\left(L^{q}(I)\right)}=\left\|\left(\int_{I}|u(\cdot, t)|^{q} d t\right)^{1 / q}\right\|_{L^{p}\left(\mathbb{R}^{d}\right)}
$$

in $L^{p}\left(\mathbb{R}^{d} ; L^{q}(I)\right)$, with the usual modification $\|u\|_{L^{p}\left(L^{\infty}(I)\right)}=\left\|\sup _{t \in I}|u(\cdot, t)|\right\|_{p}$ if $q=\infty$.

Theorem 6.1. Let $a \in(1, \infty)$ and let $I$ be a compact interval of time. Then

$$
\left\|U^{a} f\right\|_{L^{p}\left(L^{q}(I)\right)} \lesssim\|f\|_{L_{s}^{p},}, \frac{s}{a}=d\left(\frac{1}{2}-\frac{1}{p}\right)-\frac{1}{q}
$$

holds true in each of the following three cases: 
(i) $d=1,4<p<\infty, \frac{2 p}{p-2}<q \leq \infty$.

(ii) $d \geq 2, \frac{2(d+3)}{d+1}<p \leq \frac{2(d+2)}{d}, \frac{2 p}{(d+1) p-2(d+2)}<q \leq \infty$.

(iii) $d \geq 2, \frac{2(d+2)}{d}<p<\infty, 2 \leq q \leq \infty$.

Remark. The statements (i) and (iii) also hold for $0<a<1$.

Proof. The stated results for $p \leq q \leq \infty$ are in [29]. Consider the inequality

$$
\sup _{k \in \mathbb{N}} 2^{-k a\left(\frac{d}{2}-\frac{d}{p}-\frac{1}{q}\right)}\left\|U^{a}\left[P_{k} f\right]\right\|_{L^{p}\left(L^{q}(I)\right)} \lesssim\|f\|_{L^{p}}
$$

which holds for $2(d+3) /(d+1)<p \leq q \leq \infty, d \geq 2$ and $4<p \leq q \leq \infty, d=1$, by [29]. It holds for $q=2$ if $p=\infty, d=1$ and $2+4 / d<p \leq \infty$ if $d \geq 2$, by Theorem 1.4. By complex interpolation (55) also holds for $d \geq 2, \frac{2(d+3)}{d+1}<p \leq \frac{2(d+2)}{d}$ and $\frac{1}{q}<\frac{d+1}{2}-\frac{d+2}{p}$ which is equivalent with $\frac{2 p}{(d+1) p-2(d+2)}<q \leq \infty$. Moreover for $d=1$ complex interpolation shows that (55) holds for $\frac{2}{q}<1-\frac{2}{p}$ (i.e. $\frac{2 p}{p-2}<q \leq \infty$ ). Finally we may combine the dyadic pieces by using Proposition A.3 in the appendix.

Remark. For $a=2$, we obtain further improvements in [25], in particular in two dimensions an $L^{p}\left(L^{4}\right)$ bound for $p>16 / 5$.

\section{Appendix A. Combining Frequency localized Pieces}

We state a variant of results by Fefferman and Stein [17] and Miyachi [26] which is motivated by its application to prove Theorems 1.4 and 6.1. The approach extends and somewhat simplifies the one in [29] (see also [33], 28] for related results). For later applications in 25] we formulate the results in slightly more generality than needed in this paper (in particular here we just need the case $p=v$ in Theorem A.1.

Let $\mathcal{B}$ be a Banach space with norm $|\cdot|_{\mathcal{B}}$; in our application $\mathcal{B}=L^{q}(I)$ for a compact interval $I$. We consider convolution operators $T_{k}$, with $k \in \mathbb{N}$, mapping $L^{1}\left(\mathbb{R}^{d}\right)$ into $L^{1}\left(\mathbb{R}^{d}, \mathcal{B}\right)$, a space of $\mathcal{B}$-valued functions. We define $T_{k}$ by

$$
T_{k} f(x)=h_{k} * f(x)=\int h_{k}(x-y) f(y) d y,
$$

where for each $k$ we make, for simplicity, the a-priori assumption that $h_{k} \in L^{1}\left(\mathbb{R}^{d}, \mathcal{B}\right)$ but we do not assume a bound on these $L^{1}$ norms. We shall be interested in situations where, for some $a>0$, the part of the kernel $h_{k}$ supported in $|x| \geq C_{1} 2^{k(a-1)}$ can be neglected. In particular, this is true of $U_{t}^{a}$ as defined in (3).

In what follows let $\rho_{k} \in C^{1}\left(\mathbb{R}^{d}\right)$ be such that

$$
\begin{gathered}
\left|\rho_{k}(x)\right|+2^{-k}\left|\nabla \rho_{k}(x)\right| \leq 2^{k d}, \\
\operatorname{supp} \rho_{k} \subset\left\{x:|x| \leq 2^{-k}\right\} .
\end{gathered}
$$

We define $R_{k}$ on $\mathcal{B}$-valued functions $g$ by

$$
R_{k} g(x)=\rho_{k} * g(x)=\int \rho_{k}(y) g(x-y) d y .
$$

In applications the operators $R_{k}$ often arise from dyadic frequency decompositions, however no cancellation condition on $\rho_{k}$ is needed in the following result. 
Theorem A.1. Let $v_{0} \in(1, \infty), v_{0} \leq p_{0}, 1 / v_{0}-1 / p_{0}=1 / v_{1}$ and $a \in(0, \infty)$. With $T_{k}$ and $R_{k}$ defined as above, let

$$
A:=\sup _{k>0} 2^{k a d / p_{0}}\left\|T_{k}\right\|_{L^{v_{0}} \rightarrow L^{p_{0}}(\mathcal{B})}
$$

and, for $1 / v_{1}+1 / v_{1}^{\prime}=1$,

$$
B:=\sup _{k>0} 2^{k a d / p_{0}}\left(\int_{|x| \geq C_{1} 2^{k(a-1)}}\left|h_{k}(x)\right|_{\mathcal{B}}^{v_{1}} d x\right)^{1 / v_{1}^{\prime}}
$$

for some fixed constant $C_{1} \geq 1$. Then for all $p \in\left(p_{0}, \infty\right)$ and $r>0$, there exists $C=$ $C\left(p_{0}, p, r, C_{0}, C_{1}, d\right)$ so that

$$
\left\|\left(\sum_{k>0} 2^{k a d r / p}\left|R_{k} T_{k} f_{k}\right|_{\mathcal{B}}^{r}\right)^{1 / r}\right\|_{p} \leq C A\left(1+\frac{B}{A}\right)^{1-p_{0} / p}\left(\sum_{k>0}\left\|f_{k}\right\|_{v}^{p}\right)^{1 / p}, \quad \frac{1}{v}-\frac{1}{p}=\frac{1}{v_{0}}-\frac{1}{p_{0}} .
$$

Moreover,

$$
\left\|\left(\sum_{k>0}\left|R_{k} T_{k} f_{k}\right|_{\mathcal{B}}^{r}\right)^{1 / r}\right\|_{B M O} \leq C(A+B) \sup _{k>0}\left\|f_{k}\right\|_{v_{1}} .
$$

Before we begin with the proof we state a preliminary lemma.

Lemma A.2. Define

$$
\mathcal{T}_{k} f(x)=2^{k d} \int_{|x-y| \leq 2^{-k}}\left|T_{k} f(y)\right|_{\mathcal{B}} d y
$$

and, with the notation as in (58) and (59), let

$$
\mathcal{A}(p)=C_{1}^{d\left(1 / p_{0}-1 / p\right)} A+A^{p_{0} / p} B^{1-p_{0} / p} .
$$

Then, for $p_{0} \leq p \leq \infty$,

$$
\left\|\mathcal{T}_{k} f\right\|_{L^{p}(\mathcal{B})} \lesssim 2^{-k a d / p} \mathcal{A}(p)\|f\|_{v} \quad \frac{1}{v}-\frac{1}{p}=\frac{1}{v_{0}}-\frac{1}{p_{0}}
$$

Proof. We interpolate between $p=p_{0}$ and $p=\infty$. Since $\mathcal{A}\left(p_{0}\right)=A$ the inequality is immediate for $p=p_{0}$ from assumption (58).

To prove the inequality for $p=\infty$ we choose a grid $\mathfrak{Q}_{a}^{k}$ of cubes $\mathcal{Q}$ of sidelength $2^{k(a-1)}$, so that the cubes in $\mathfrak{Q}_{a}^{k}$ have disjoint interior and $\sum_{\mathcal{Q} \in \mathfrak{Q}_{a}^{k}} \chi_{\mathcal{Q}}=1$ almost everywhere. For each $\mathcal{Q} \in \mathfrak{Q}_{a}^{k}$ let $\mathcal{Q}^{*}$ be the cube with same center as $\mathcal{Q}$ and sidelength $10 d C_{1} 2^{k(a-1)}$. It then suffices to show that for each cube $\mathcal{Q} \in \mathfrak{Q}_{a}^{k}$

$$
\chi_{\mathcal{Q}}(x)\left|\mathcal{T}_{k} f(x)\right|_{\mathcal{B}} \lesssim\left(C_{1}^{d / p_{0}} A+B\right)\|f\|_{\infty}
$$

for every $x$. Given $\mathcal{Q}$ we split $f=b_{\mathcal{Q}}+g_{\mathcal{Q}}$ where $b_{\mathcal{Q}}=f \chi_{\mathcal{Q}^{*}}$ and $g_{\mathcal{Q}}=f \chi_{\mathbb{R}^{d} \backslash \mathcal{Q}^{*}}$. For $b_{\mathcal{Q}}$ we apply Hölder's inequality and use assumption (58), so that

$$
\begin{aligned}
\left|\mathcal{T}_{k} b_{\mathcal{Q}}(x)\right|_{\mathcal{B}} & \lesssim 2^{k d / p_{0}}\left\|\left|T_{k} b_{\mathcal{Q}}\right|_{\mathcal{B}}\right\|_{p_{0}} \lesssim 2^{k d / p_{0}} A 2^{-k a d / p_{0}}\left\|b_{\mathcal{Q}}\right\|_{v_{0}} \\
& \lesssim A 2^{k d / p_{0}} 2^{-k a d / p_{0}}\left|\mathcal{Q}^{*}\right|^{1 / v_{0}-1 / v_{1}}\|f\|_{v_{1}} \lesssim C(d) C_{1}^{d / v_{0}-d / v_{1}} A\|f\|_{v_{1}}
\end{aligned}
$$

since $\left|\mathcal{Q}^{*}\right| \approx C_{1}^{d} 2^{k(a-1) d}$. 
For $g_{\mathcal{Q}}$ we note that when $x \in \mathcal{Q}, w \notin \mathcal{Q}^{*}$ and $|x-z| \leq 2^{-k}$, then $|z-w| \geq C_{1} 2^{k(a-1)}$, so we can use (59) to estimate

$$
\begin{aligned}
\left|\mathcal{T}_{k} g_{\mathcal{Q}}(x)\right|_{\mathcal{B}} & \leq 2^{k d} \int_{|x-z| \leq 2^{-k}} \int_{|z-w| \geq C_{1} 2^{k(a-1)}}\left|h_{k}(z-w)\right|_{\mathcal{B}}|f(w)| d w d z \\
& \lesssim B\|f\|_{v_{1}}
\end{aligned}
$$

Combining the two estimates, we get (63).

Proof of Theorem A.1. We may assume $r \leq 1$ and that the summation in $k$ is extended over a finite set. We proceed as in [28] and, by the Fefferman-Stein theorem [17] on the \#-maximal operator and the inequality $\left.|| u\right|_{\mathcal{B}} ^{r}-|v|_{\mathcal{B}}^{r}|\leq| u-\left.v\right|_{\mathcal{B}} ^{r}$ we get

$$
\begin{aligned}
& \left\|\left(\sum_{k}\left|2^{k a d / p} R_{k} T_{k} f_{k}\right|_{\mathcal{B}}^{r}\right)^{1 / r}\right\|_{p} \\
& \lesssim\left\|\sup _{Q: x \in Q} \sum_{k} 2^{k a d r / p} \int_{Q} \int_{Q}\left|R_{k} T_{k} f_{k}(y)-R_{k} T_{k} f_{k}(z)\right|_{\mathcal{B}}^{r} d z d y\right\|_{L^{p / r}(d x)}^{1 / r}
\end{aligned}
$$

Let $x \mapsto Q(x)$ depend measurably on $x$, so that for each $x$ the cube $Q(x)$ is centered at $x$ and has sidelength in $\left[2^{L(x)}, 2^{L(x)+1}\right)$. It suffices to estimate the $L^{p}$ norm of

$$
\left(\sum_{k} 2^{k a d r / p} \int_{Q(x)} \int_{Q(x)}\left|R_{k} T_{k} f_{k}(y)-R_{k} T_{k} f_{k}(z)\right|_{\mathcal{B}}^{r} d z d y\right)^{1 / r} .
$$

We let $F=\left\{f_{k}\right\}_{k>0}$ and estimate the displayed expression by $\sum_{i=1}^{3} \mathfrak{S}_{i} F(x)$ where

$$
\begin{aligned}
& \mathfrak{S}_{1} F(x)=\left(\sum_{k+L(x) \leq 0} 2^{k a d r / p} \int_{Q(x)} \int_{Q(x)}\left|R_{k} T_{k} f_{k}(y)-R_{k} T_{k} f_{k}(z)\right|_{\mathcal{B}}^{r} d z d y\right)^{1 / r}, \\
& \mathfrak{S}_{2} F(x)=\left(\sum_{\substack{k+L(x)>0 \\
k(a-1) \leq L(x)}} 2^{k a d r / p} \int_{Q(x)}\left|R_{k} T_{k} f_{k}(y)\right|_{\mathcal{B}}^{r} d y\right)^{1 / r}, \\
& \mathfrak{S}_{3} F(x)=\left(\sum_{\substack{k+L(x)>0 \\
k(a-1)>L(x)}} 2^{k a d r / p} \int_{Q(x)}\left|R_{k} T_{k} f_{k}(y)\right|_{\mathcal{B}}^{r} d y\right)^{1 / r} .
\end{aligned}
$$

Set $\|F\|_{\ell^{p}\left(L^{v}\right)}=\left(\sum_{k}\left\|f_{k}\right\|_{v}^{p}\right)^{1 / p}$ for $p<\infty$ and $\|F\|_{\ell^{\infty}\left(L^{v_{1}}\right)}=\sup _{k}\left\|f_{k}\right\|_{v_{1}}$. For $p>p_{0}$ we will bound the $L^{p}$ norms of $\mathfrak{S}_{i} F$ by $C \mathcal{A}(p)\|F\|_{\ell^{p}\left(L^{v}\right)}$. In the proofs we shall use the notation

$$
\omega_{k}(x)=2^{k d} \chi_{\left\{|x| \leq \sqrt{d} 2^{-k+3}\right\}}(x) .
$$

$L^{p}$ bound for $\mathfrak{S}_{1}(F)$. Using the estimate (56) for $\nabla \rho_{k}$ we see that for $y, z \in Q(x)$

$$
\left|R_{k} T_{k} f_{k}(y)-R_{k} T_{k} f_{k}(z)\right|_{\mathcal{B}} \lesssim 2^{L(x)+k} \int \omega_{k}(x-u)\left|T_{k} f_{k}(u)\right|_{\mathcal{B}} d u .
$$

Using the embedding $\ell^{p / r} \hookrightarrow \ell^{\infty}$ we estimate

$$
\begin{aligned}
\mathfrak{S}_{1} F(x)^{r} & \lesssim \sup _{L}\left[\sum_{k+L \leq 0} 2^{k+L} \int \omega_{k}(x-u)\left|2^{k a d / p} T_{k} f_{k}(u)\right|_{\mathcal{B}} d u\right]^{r} \\
& \lesssim\left(\sum_{L}\left[\sum_{0<k \leq-L} 2^{k+L} \int \omega_{k}(x-u)\left|2^{k a d / p} T_{k} f_{k}(u)\right|_{\mathcal{B}} d u\right]^{p}\right)^{r / p}
\end{aligned}
$$


and therefore, with the change of summation variable $n=-L-k$ and Minkowski's inequality

$$
\begin{aligned}
& \left\|\mathfrak{S}_{1} F\right\|_{p}=\left\|\left[\mathfrak{S}_{1} F\right]^{r}\right\|_{p / r}^{1 / r} \\
& \lesssim \sum_{n>0} 2^{-n}\left(\sum_{L>-n}\left\|2^{-(L+n) d} \int_{|y| \leq C 2^{L+n}} 2^{-(L+n) a d / p}\left|T_{-L-n} f_{-L-n}(\cdot-y)\right|_{\mathcal{B}} d y\right\|_{p}^{p}\right)^{1 / p} .
\end{aligned}
$$

By Lemma A.2 this can be estimated by

$$
\mathcal{A}(p) \sum_{n>0} 2^{-n}\left(\sum_{L>-n}\left\|f_{-L-n}\right\|_{v}^{p}\right)^{1 / p} \lesssim \mathcal{A}(p)\|F\|_{\ell^{p}\left(L^{v}\right)}
$$

$L^{p}$ bound for $\mathfrak{S}_{2}(F)$. The $L^{p}$ bound for $\mathfrak{S}_{2} F$ follows by interpolating the inequalities

$$
\begin{aligned}
&\left\|\mathfrak{S}_{2} F\right\|_{p_{0}} \lesssim A\|F\|_{\ell^{p_{0}}\left(L^{v_{0}}\right)}, \\
&\left\|\mathfrak{S}_{2} F\right\|_{\infty} \lesssim\left(C_{1}^{d / p_{0}} A+B\right)\|F\|_{\ell^{\infty}\left(L^{v_{1}}\right)} .
\end{aligned}
$$

For the $L^{p_{0}}$ bound, we sum a geometric series, using $p>p_{0}$, to estimate

$$
\left(\int_{Q(x)} \sum_{\substack{k+L(x)>0 \\ k(a-1) \leq L(x)}} 2^{k a d r / p}\left|R_{k} T_{k} f_{k}(y)\right|_{\mathcal{B}}^{r} d y\right)^{1 / r} \lesssim\left(M\left[\sup _{k>0} 2^{k a d r / p_{0}}\left|R_{k} T_{k} f_{k}\right|_{\mathcal{B}}^{r}\right](x)\right)^{1 / r}
$$

and by the $L^{p_{0} / r}$ boundedness of the Hardy-Littlewood operator we get

$$
\begin{aligned}
\left\|\mathfrak{S}_{2} F\right\|_{p_{0}} & \lesssim\left\|\sup _{k>0} 2^{k a d r / p_{0}}\left|R_{k} T_{k} f_{k}\right|_{\mathcal{B}}^{r}\right\|_{p_{0} / r}^{1 / r}=\left\|\sup _{k>0} 2^{k a d / p_{0}}\left|R_{k} T_{k} f_{k}\right|_{\mathcal{B}}\right\|_{p_{0}} \\
& \lesssim\left(\sum_{k>0}\left\|2^{k a d / p_{0}}\left|R_{k} T_{k} f_{k}\right|_{\mathcal{B}}\right\|_{p_{0}}^{p_{0}}\right)^{1 / p_{0}} \lesssim\left(\sum_{k>0}\left\|2^{k a d / p_{0}}\left|T_{k} f_{k}\right|_{\mathcal{B}}\right\|_{p_{0}}^{p_{0}}\right)^{1 / p_{0}} \\
& \lesssim A\|F\|_{\ell^{p_{0}\left(L^{v_{0}}\right)}},
\end{aligned}
$$

For the $L^{\infty}$ bound, we fix $x, Q=Q(x), L=L(x)$ and let $y_{Q}=y_{Q(x)}$ be the center of $Q$. By Hölder's inequality and (56),

$$
\left.\mathfrak{S}_{2} F(x) \lesssim\left(\left.\sum_{\substack{k+L>0 \\ k(a-1) \leq L}} 2^{k a d r / p}\left[\int_{Q} \int \omega_{k}(y-z) \mid T_{k} f_{k}\right](z)\right|_{\mathcal{B}} d z d y\right]^{r}\right)^{1 / r} .
$$

Let $Q^{*}$ be the $C_{1} 2^{10} d$ dilate of $Q$ with respect to $y_{Q}$. We may estimate the last displayed expression by $\mathcal{E}^{\text {near }}+\mathcal{E}^{\text {far }}$ where

$$
\begin{aligned}
\mathcal{E}^{\text {near }} & =\left(\sum_{\substack{k+L>0 \\
k(a-1) \leq L}} 2^{k a d r / p}\left[\int_{Q} \int \omega_{k}(y-z)\left|T_{k}\left[f_{k} \chi_{Q^{*}}\right](z)\right|_{\mathcal{B}} d z d y\right]^{r}\right)^{1 / r}, \\
\mathcal{E}^{\text {far }} & =\left(\sum_{\substack{k+L>0 \\
k(a-1) \leq L}} 2^{k a d r / p}\left[\int_{Q} \int \omega_{k}(y-z)\left|T_{k}\left[f_{k} \chi_{\mathbb{R}^{d} \backslash Q^{*}}\right](z)\right|_{\mathcal{B}} d z d y\right]^{r}\right)^{1 / r},
\end{aligned}
$$

and it suffices to check that

$$
\begin{aligned}
\mathcal{E}^{\text {near }} & \lesssim C_{1}^{d / p_{0}} A\|F\|_{\ell^{\infty}\left(L^{v_{1}}\right)} \\
\mathcal{E}^{\text {far }} & \lesssim B\|F\|_{\ell^{\infty}\left(L^{v_{1}}\right)} .
\end{aligned}
$$


To prove (65) we apply Hölder's inequality, use $p_{0}<p$ and assumption (58):

$$
\begin{aligned}
\mathcal{E}^{\text {near }} & \lesssim\left(\sum_{k} 2^{k a d r / p}\left(\frac{C_{1}^{d}}{\left|Q^{*}\right|} \int\left|T_{k}\left[f_{k} \chi_{Q^{*}}\right](z)\right|_{\mathcal{B}}^{p_{0}} d z\right)^{r / p_{0}}\right)^{1 / r} \\
& \lesssim C_{1}^{d / p_{0}} \sup _{k} 2^{k a d / p_{0}}\left|Q^{*}\right|^{-1 / p_{0}}\left\|T_{k}\left[f_{k} \chi_{Q^{*}}\right]\right\|_{L^{p_{0}(\mathcal{B})}} \\
& \lesssim A C_{1}^{d / p_{0}} \sup _{k}\left|Q^{*}\right|^{-1 / p_{0}}\left\|f_{k} \chi_{Q^{*}}\right\|_{v_{0}} \lesssim A C_{1}^{d / p_{0}} \sup _{k}\left|Q^{*}\right|^{-1 / p_{0}}\left|Q^{*}\right|^{1 / v_{0}-1 / v_{1}}\left\|f_{k} \chi_{Q^{*}}\right\|_{v_{1}} \\
& \lesssim A C_{1}^{d / p_{0}}\|F\|_{\ell^{\infty}\left(L^{v_{1}}\right)} .
\end{aligned}
$$

To prove (66) we use assumption (59). Note that if $y \in Q,|y-z| \leq \sqrt{d} 2^{-k+3}$ (and $k(a-1) \leq L), w \in \mathbb{R}^{d} \backslash Q^{*}$, then $|z-w| \geq C_{1} 2^{k(a-1)}$. Thus

$$
\begin{aligned}
& \int_{Q} \int \omega_{k}(y-z)\left|T_{k}\left[f_{k} \chi_{\mathbb{R}^{d} \backslash Q^{*}}\right](z)\right|_{\mathcal{B}} d z d y \\
& \lesssim \int_{Q} \int \omega_{k}(y-z) \int_{|z-w| \geq C_{1} 2^{k(a-1)}}\left|h_{k}(z-w)\right|_{\mathcal{B}}\left|f_{k}(w)\right| d w d z d y \\
& \lesssim 2^{-k a d / p_{0}} B\|F\|_{\ell^{\infty}\left(L^{v_{1}}\right)}
\end{aligned}
$$

and (66) follows.

$L^{p}$ bound for $\mathfrak{S}_{3}(F)$. Let $B_{L}$ be the ball of radius $10 d 2^{L}$ centered at the origin. We may estimate

$$
\begin{aligned}
\left\|\mathfrak{S}_{3}(F)\right\|_{p} & \lesssim\left\|\sup _{L} \frac{\chi_{B_{L}}}{\left|B_{L}\right|} *\left[\sum_{\substack{k+L>0 \\
k(a-1)>L}} 2^{k a d r / p}\left|R_{k} T_{k} f_{k}\right|_{\mathcal{B}}^{r}\right]\right\|_{p / r}^{1 / r} \\
& \lesssim\left(\sum_{n>0}\left\|\sup _{L<\left(1-a^{-1}\right) n} \frac{\chi_{B_{L}}}{\left|B_{L}\right|} *\left[2^{(n-L) a d r / p}\left|R_{n-L} T_{n-L} f_{n-L}\right|_{\mathcal{B}}^{r}\right]\right\|_{p / r}\right)^{1 / r}
\end{aligned}
$$

by Minkowski's inequality. By Hölder's inequality on each ball $B_{L}$ we see that the last expression is dominated by

$$
\left(\sum_{n>0}\left\|\sup _{L<\left(1-a^{-1}\right) n} \frac{\chi_{B_{L}}}{\left|B_{L}\right|} * 2^{(n-L) a d / p}\left|R_{n-L} T_{n-L} f_{n-L}\right|_{\mathcal{B}}\right\|_{p}^{r}\right)^{1 / r} .
$$

Now for $n>0$ we have $\chi_{B_{L}} * \omega_{n-L}(x) \lesssim \chi_{B_{L+1}}(x)$. Thus we get

$$
\left\|\mathfrak{S}_{3}(F)\right\|_{p} \lesssim\left(\sum_{n>0}\left\|\mathfrak{S}_{3, n} F\right\|_{p}^{r}\right)^{1 / r}
$$

where

$$
\mathfrak{S}_{3, n} F(x)=\sup _{L<\left(1-a^{-1}\right) n} 2^{-L d} \chi_{B_{L+1}} * 2^{(n-L) a d / p}\left|T_{n-L} f_{n-L}\right|_{\mathcal{B}}
$$

It thus suffices to prove

$$
\left\|\mathfrak{S}_{3, n} F\right\|_{p} \lesssim 2^{-n d\left(\frac{1}{p_{0}}-\frac{1}{p}\right)} \mathcal{A}(p)\|F\|_{\ell^{p}\left(L^{v}\right)} .
$$

We shall use an analytic interpolation argument and for this it is necessary to linearize the operator. For any bounded linear functional $\lambda \in \mathcal{B}^{*}$ we denote by $\langle v, \lambda\rangle$ the action of $\lambda$ on $v \in \mathcal{B}$. Let $(x, y) \rightarrow u_{L}(x, y)$ be any measurable function with values in $\mathcal{B}^{*}$, so that 
$\left\|u_{L}\right\|_{\infty} \leq 1$. After replacing a sup in $L$ by an $\ell^{p}$ norm and interchanging an integral and a summation it then suffices to bound

$$
\left(\sum_{L<\left(1-a^{-1}\right) n}\left\|2^{(n-L) a d / p} 2^{-L d} \int \chi_{B_{L+1}}(y)\left\langle T_{n-L} f_{n-L}(\cdot-y), u_{L}(\cdot, y)\right\rangle d y\right\|_{p}^{p}\right)^{1 / p}
$$

by the right hand side of (67), with a constant uniform in the choices of the $u_{L}$. In what follows we fix such a choice.

Define an analytic family

$$
\mathcal{G}_{L, n}^{z} F(x)=2^{(n-L) a d(1-z) / p_{0}} 2^{-L d} \int \chi_{B_{L+1}}(y)\left\langle T_{n-L} f_{n-L}(x-y), u_{L}(x, y)\right\rangle d y .
$$

We then show that for $p_{0} \leq \widetilde{p} \leq \infty$

$$
\begin{aligned}
\left(\sum_{L<\left(1-a^{-1}\right) n}\left\|\mathcal{G}_{L, n}^{z} F\right\|_{\widetilde{p}}^{\widetilde{p}}\right)^{1 / \widetilde{p}} \lesssim 2^{-n d\left(\frac{1}{p_{0}}-\frac{1}{\tilde{p}}\right)} \mathcal{A}(\widetilde{p})\|F\|_{\ell \widetilde{p}\left(L^{\widetilde{v}}\right)} & \\
& (1-\operatorname{Re}(z))\left(\frac{1}{v_{0}}, \frac{1}{p}\right)+\operatorname{Re}(z)\left(\frac{1}{v_{1}}, \frac{1}{\infty}\right)=\left(\frac{1}{\widetilde{v}}, \frac{1}{\widetilde{p}}\right)
\end{aligned}
$$

and the required $L^{p}$ estimate follows if we let $z=\left(1-p_{0} / p\right)$. By Stein's theorem on analytic families of operators it suffices to show (68) for $\operatorname{Re}(z)=0, \widetilde{p}=p_{0}$ and $\operatorname{Re}(z)=1, \widetilde{p}=\infty$.

First, for $\widetilde{p}=p_{0}, z=\imath \gamma$ we bound

$$
\begin{aligned}
& \left(\sum_{L<\left(1-a^{-1}\right) n}\left\|\mathcal{G}_{L, n}^{\imath \gamma} F\right\|_{p_{0}}^{p_{0}}\right)^{1 / p_{0}} \lesssim\left(\sum_{L<\left(1-a^{-1}\right) n} 2^{(n-L) a d}\left\|T_{n-L} f_{n-L}\right\|_{L^{p_{0}(\mathcal{B})}}^{p_{0}}\right)^{1 / p_{0}} \\
& \lesssim A\left(\sum_{L<n}\left\|f_{n-L}\right\|_{v_{0}}^{p_{0}}\right)^{1 / p_{0}} \lesssim A\|F\|_{\ell^{p_{0}}\left(L^{v_{0}}\right)}
\end{aligned}
$$

which is 68 for $\operatorname{Re}(z)=0$.

Now let $\widetilde{p}=\infty, \operatorname{Re}(z)=1$. The required bound for $\mathcal{G}_{L, n}^{1+\imath \gamma}$ follows if we can show that for any fixed $x_{0}$ and fixed $L<\left(1-a^{-1}\right) n$

$$
2^{-L d} \chi_{B_{L+1}} *\left|T_{n-L} f_{n-L}\right|_{\mathcal{B}}\left(x_{0}\right) \lesssim 2^{-n d / p_{0}}\left(C_{1}^{d / p_{0}} A+B\right)\|F\|_{\ell^{\infty}\left(L^{v_{1}}\right)} .
$$

Let $\mathcal{Q}^{*}$ be a cube of sidelength $20 d C_{1} 2^{(n-L)(a-1)}$ centered at $x_{0}$; recall the inequality $(n-L)(a-1)>L$. We dominate the left hand side of (69) by $C\left(\mathcal{E}_{n}^{\text {near }}+\mathcal{E}_{n}^{\text {far }}\right)$, where

$$
\begin{aligned}
\mathcal{E}_{n}^{\text {near }} & =\int 2^{-L d} \chi_{B_{L+1}}\left(x_{0}-z\right)\left|T_{n-L}\left[f_{n-L} \chi_{\mathcal{Q}^{*}}\right](z)\right|_{\mathcal{B}} d z, \\
\mathcal{E}_{n}^{\text {far }} & =\int 2^{-L d} \chi_{B_{L+1}}\left(x_{0}-z\right)\left|T_{n-L}\left[f_{n-L} \chi_{\mathbb{R}^{d} \backslash \mathcal{Q}^{*}}\right](z)\right|_{\mathcal{B}} d z .
\end{aligned}
$$

By Hölder's inequality

$$
\begin{aligned}
\mathcal{E}_{n}^{\text {near }} & \lesssim\left(2^{-L d} \int\left|T_{n-L}\left[f_{n-L} \chi_{\mathcal{Q}^{*}}\right](z)\right|_{\mathcal{B}}^{p_{0}} d z\right)^{1 / p_{0}} \\
& \lesssim A 2^{-(n-L) a d / p_{0}} 2^{-L d / p_{0}}\left\|f_{n-L} \chi_{\mathcal{Q}^{*}}\right\|_{v_{0}}
\end{aligned}
$$

and since $\left\|f_{n-L} \chi \mathcal{Q}^{*}\right\|_{v_{0}} \lesssim C_{1}^{d / p_{0}} 2^{(n-L)(a-1) d / p_{0}}\left\|f_{n-L}\right\|_{v_{1}}$ this yields

$$
\mathcal{E}_{n}^{\text {near }} \lesssim C_{1}^{d / p_{0}} A 2^{-n d / p_{0}}\|F\|_{\ell^{\infty}\left(L^{v_{1}}\right)} .
$$


Next observe that if $x_{0}-z \in B_{L+1}$ and $y \in \mathbb{R}^{d} \backslash \mathcal{Q}^{*}$ then $|z-y| \geq C_{1} 2^{(n-L)(a-1)}$ and thus

$$
\begin{aligned}
\mathcal{E}_{n}^{\mathrm{far}} & \lesssim \int 2^{-L d} \chi_{B_{L+1}}\left(x_{0}-z\right) \int_{|z-y| \geq C_{1} 2^{(n-L)(a-1)}}\left|h_{n-L}(z-y)\right|_{\mathcal{B}}\left|f_{n-L}(y)\right| d y d z \\
& \lesssim 2^{-(n-L) a d / p_{0}} B\left\|f_{n-L}\right\|_{v_{1}} .
\end{aligned}
$$

Since by assumption $a L<(a-1) n$ we get

$$
\mathcal{E}_{n}^{\mathrm{far}} \lesssim B 2^{-n d / p_{0}}\|F\|_{\ell^{\infty}\left(L^{v_{1}}\right)}
$$

The estimates for $\mathcal{E}_{n}^{\text {near }}$ and $\mathcal{E}_{n}^{\text {far }}$ yield (69). This finishes the estimation of $\mathfrak{S}_{3, n}(F)$ and thus concludes the proof of the theorem.

Applications. We show how for the semigroups $U_{t}^{a}$ one can use Theorem A.1 to prove global estimates from frequency localized versions.

Let $\varphi \in C^{\infty}(\mathbb{R})$ be supported in $(1 / 4,4)$ and not identically zero. Define $s_{\mathrm{cr}}=s_{a \text { cr }}$ by

$$
s_{\mathrm{cr}}(v ; p, q):=d\left(\frac{1}{v}-\frac{1}{p}\right)+a\left(\frac{d}{2}-\frac{d}{v}-\frac{1}{q}\right)
$$

which, for a large range of parameters, turns out to be a critical for $L_{s}^{v} \rightarrow L^{p}\left(\mathbb{R}^{d} ; L^{q}(I)\right)$ estimates; in particular $s_{\mathrm{cr}}(p ; p, q)=a\left(\frac{d}{2}-\frac{d}{p}-\frac{1}{q}\right)$. Let

$$
\Gamma_{a}(v ; p, q):=\sup _{R>1} R^{s_{\mathrm{cr}}(v ; p, q)}\left\|U^{a} \varphi\left(R^{-1}|D|\right)\right\|_{L^{v} \rightarrow L^{p}\left(\mathbb{R}^{d} ; L^{q}[-1 / 2,1 / 2]\right)} .
$$

Clearly this definition depends on $\varphi$, however the finiteness of $\Gamma_{a}(v ; p, q)$ is independent of the particular $\varphi$ used.

Proposition A.3. Let $v_{0}, p_{0}, q_{0} \in[1, \infty], p \in\left(p_{0}, \infty\right), q \geq q_{0}, r \in(0, \infty), v_{0} \leq p_{0}$, and let $I$ be a compact interval. Suppose that $\Gamma_{a}\left(v_{0} ; p_{0}, q_{0}\right)$ is finite and let $s_{\mathrm{cr}}$ be as in (70). Assume that $1 / v_{0}-1 / p_{0}=1 / v-1 / p$. Then:

$$
\left\|\left(\sum_{k>0}\left(\int_{I}\left|P_{k} U^{a} f(\cdot, t)\right|^{q} d t\right)^{r / q}\right)^{1 / r}\right\|_{L^{p}\left(\mathbb{R}^{d}\right)} \lesssim\left(\sum_{k>0} 2^{k s p}\left\|P_{k} f\right\|_{v}^{p}\right)^{1 / p}, \quad s=s_{\mathrm{cr}}(v ; p, q),
$$

and

$$
\left\|\left(\int_{I}\left|U^{a} f(\cdot, t)\right|^{q} d t\right)^{1 / q}\right\|_{L^{p}\left(\mathbb{R}^{d}\right)} \lesssim\|f\|_{B_{s, p}^{v}\left(\mathbb{R}^{d}\right)}, \quad s=s_{\mathrm{cr}}(v ; p, q) .
$$

(ii) If $1 / v_{0}-1 / p_{0}=1 / v_{1}$ then

$$
\left\|\left(\sum_{k>0}\left(\int_{I}\left|P_{k} U^{a} f(\cdot, t)\right|^{q} d t\right)^{r / q}\right)^{1 / r}\right\|_{B M O\left(\mathbb{R}^{d}\right)} \lesssim \sup _{k>0} 2^{k s}\left\|P_{k} f\right\|_{v_{1}}, \quad s=s_{\mathrm{cr}}\left(v_{1} ; \infty, q\right) .
$$

(iii) If $t \mapsto \varpi(t)$ is smooth and compactly supported then

$$
\left\|\varpi U^{a} f\right\|_{L^{p}\left(\mathbb{R}^{d} ; B_{\gamma, r}^{q}(\mathbb{R})\right)} \lesssim\|f\|_{B_{s+a \gamma, p}^{v}\left(\mathbb{R}^{d}\right)}, \quad s=s_{\mathrm{Cr}}(v ; p, q) .
$$

If $f \in B_{s, p}^{v}\left(\mathbb{R}^{d}\right)$ with $s=s_{\mathrm{cr}}(v ; p, \infty)$ then the function $t \mapsto U^{a} f(x, t)$ is continuous locally in $B_{1 / q, r}^{q}(\mathbb{R})$, for almost every $x \in \mathbb{R}^{d}$, and we have the maximal inequality

$$
\left\|\sup _{t \in I}\left|U^{a} f(\cdot, t)\right|\right\|_{L^{p}\left(\mathbb{R}^{d}\right)} \lesssim\|f\|_{B_{s, p}^{v}\left(\mathbb{R}^{d}\right)}, \quad s=a\left(\frac{d}{2}-\frac{d}{v}\right)+d\left(\frac{1}{v}-\frac{1}{p}\right) .
$$


We note that the constants implicit in (72) and (75) depend on $p, q, q_{0}, I, a, d, \varpi$.

For the proof of Proposition A.3 we need a standard imbedding result.

Lemma A.4. Let $v, p \in[1, \infty]$ and $1 \leq q_{0} \leq q \leq \infty$. Then

$$
\Gamma_{a}(v ; p, q) \lesssim \Gamma_{a}\left(v ; p, q_{0}\right) .
$$

Proof. Let $h$ be in $C^{1}(-1 / 2,1 / 2)$. By the fundamental theorem of calculus,

$$
|h(t)|^{q_{0}} \leq|h(\tau)|^{q_{0}}+q_{0} \int_{-1 / 2}^{1 / 2}|h(y)|^{q_{0}-1}\left|h^{\prime}(y)\right| d y .
$$

for all $t, \tau \in(-1 / 2,1 / 2)$. Integrating in $\tau \in(-1 / 2,1 / 2)$ and applying Hölder's inequality yields

$$
\sup _{-1 / 2<t<1 / 2}|h(t)|^{q_{0}} \leq\|h\|_{q_{0}}^{q_{0}}+q_{0}\|h\|_{q_{0}}^{q_{0}-1}\left\|h^{\prime}\right\|_{q_{0}}
$$

where the $L^{q}$ norms are on $(-1 / 2,1 / 2)$. Now, as $\|h\|_{q}^{q} \leq\|h\|_{\infty}^{q-q_{0}}\|h\|_{q_{0}}^{q_{0}}$, we have

$$
\|h\|_{q} \leq 2^{1 / q}\left(\|h\|_{q_{0}}+q_{0}^{\frac{1}{q_{0}}-\frac{1}{q}}\|h\|_{q_{0}}^{1-\frac{1}{q_{0}}+\frac{1}{q}}\left\|h^{\prime}\right\|_{q_{0}}^{\frac{1}{q_{0}}-\frac{1}{q}}\right) .
$$

Setting $\mathcal{U}_{R} f(x, t):=U^{a}\left[\varphi\left(R^{-1}|D|\right) f\right](x, t)$, for fixed $x$ we apply the displayed inequality with $h(t)=\mathcal{U}_{R} f(x, t)$, then integrate and apply Hölder's inequality in $x$ to get

$$
\left\|\mathcal{U}_{R} f\right\|_{L^{p}\left(L^{q}\right)} \leq 2^{\frac{1}{p}+\frac{1}{q}}\left(\left\|\mathcal{U}_{R} f\right\|_{L^{p}\left(L^{q_{0}}\right)}+q_{0}^{\frac{1}{q_{0}}-\frac{1}{q}}\left\|\mathcal{U}_{R} f\right\|_{L^{p}\left(L^{q_{0}}\right)}^{\frac{1}{q^{\gamma}}+\frac{1}{q}}\left\|\partial_{t} \mathcal{U}_{R} f\right\|_{L^{p}\left(L^{q_{0}}\right)}^{\frac{1}{q_{0}}-\frac{1}{q}}\right) .
$$

Now by definition $\partial_{t} U^{a}=\imath(-\Delta)^{a / 2} U^{a}$, so that

$$
\left\|\mathcal{U}_{R} f\right\|_{L^{p}\left(L^{q_{0}}\right)}+R^{-a}\left\|\partial_{t} \mathcal{U}_{R} f\right\|_{L^{p}\left(L^{q_{0}}\right)} \lesssim \Gamma_{a}\left(v ; p, q_{0}\right) R^{s_{\mathrm{cr}}(v ; p, q)}\|f\|_{v}
$$

and substituting these bounds into the displayed inequality implies the assertion.

Proof of Proposition A.3. We can reduce to the situation where $I=[-1 / 2,1 / 2]$ or $\varpi \epsilon$ $C_{c}^{\infty}((-1 / 2,1 / 2))$, by a change of variables argument. By Lemma A.4 we may assume $q_{0}=q$.

To prove (72) we apply Theorem A.1. Let $\rho$ be a radial $C^{\infty}\left(\mathbb{R}^{d}\right)$-function which is compactly supported in the ball of radius $1 / 2$ centered at 0 , with the property that $\hat{\rho}$ is positive on $\operatorname{supp} \chi(|\cdot|)$. Denote by $R_{k}$ the operator of convolution with $2^{k d} \rho\left(2^{k} \cdot\right)$. Let $L_{k}=\varphi\left(2^{-k}|D|\right)$ where $\varphi$ is chosen so that $\varphi(|\cdot|) \widehat{\rho}=1$ on $\operatorname{supp} \chi(|\cdot|)$. Thus $R_{k} L_{k} P_{k}=P_{k}$.

Now let $\mathcal{B}=L^{q}[-1 / 2,1 / 2]$ and let $T_{k} f(x, t)=2^{k(a-1) d\left(\frac{1}{v_{0}}-\frac{1}{p_{0}}\right)} 2^{-k a\left(\frac{d}{2}-\frac{1}{q}\right)} L_{k} U^{a} f(x, t)$. Then the hypothesis that $\Gamma_{a}\left(v_{0} ; p_{0}, q_{0}\right)$ is finite implies

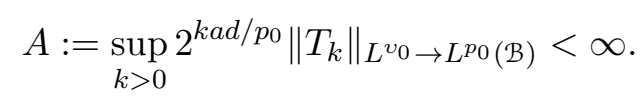

For fixed $t$ let $h_{k}^{t}$ be the convolution kernel for $T_{k}$ (at fixed time $t$ ); it can be written as

$$
h_{k}^{t}(x)=2^{k(1-a) d\left(\frac{1}{v_{0}}-\frac{1}{p_{0}}\right)} 2^{-k a\left(\frac{d}{2}-\frac{1}{q}\right)} 2^{k d}(2 \pi)^{-d} \int_{\mathbb{R}^{d}} \varphi(|\xi|) e^{\imath\left(2^{k}\langle x, \xi\rangle+2^{a k} t|\xi|^{a}\right)} d \xi .
$$

An $N$-fold integration by parts yields

$$
2^{k(a-1) d\left(\frac{1}{v_{0}}-\frac{1}{p_{0}}\right)} 2^{k a\left(\frac{d}{2}-\frac{1}{q}\right)}\left|h_{k}^{t}(x)\right| \leq C_{N} 2^{k(d-N)}|x|^{-N}, \quad|x| \geq 2^{(a-1) k+4}, \quad t \in[0,1],
$$


and thus condition (59) is satisfied with $C_{1}=2^{5}$. Thus, by Theorem A.1 we obtain the inequality

$$
\left\|\left(\sum_{k>0} 2^{k a \frac{d}{p} r}\left\|R_{k} T_{k} f_{k}\right\|_{L^{q}[-1 / 2,1 / 2]}^{r}\right)^{1 / r}\right\|_{p} \lesssim\left(\sum_{k>0}\left\|f_{k}\right\|_{v}^{p}\right)^{1 / p} .
$$

Notice that, in view of $1 / v_{0}-1 / p_{0}=1 / v-1 / p$,

$$
2^{k a d / p} T_{k}=2^{-k s_{\mathrm{cr}}(v ; p, q)} L_{k} U \text {. }
$$

Thus if we apply (77) with $f_{k}=2^{k s_{\mathrm{cr}}(v ; p, q)} P_{k} f$, then (72) follows. The assertion (74) is obtained in the same way.

We now need to show how to obtain (73) from (72). The right hand side in (72) is just the $B_{s, p}^{v}$-norm of $f$. We also have for $1<q, p<\infty$

$$
\left\|\left(\int_{I}|G(\cdot, t)|^{q} d t\right)^{1 / q}\right\|_{p} \lesssim\left\|\left(\int_{I}\left[\left(\sum_{k \geq 0}\left|P_{k} G(\cdot, t)\right|^{2}\right)^{1 / 2}\right]^{q} d t\right)^{1 / q}\right\|_{p}
$$

which we apply for $G(x, t)=U f(x, t)$. For $p=q$ this is just a consequence of the standard Littlewood-Paley inequality (after interchanging the $x$ and the $t$ integral). By CalderónZygmund theory the estimate also holds for $1<p<q$ (and is obtained by interpolation with a weak-type $(1,1)$ estimate for $L^{q}\left(\ell^{2}\right)$-valued functions). If we dualize a similar reasoning yields the case $q>p$. Inequalities (72), (78) easily imply (73).

Now consider a standard inhomogeneous Littlewood-Paley decomposition $\left\{\mathcal{P}_{k}\right\}_{k=0}^{\infty}$ on $L^{p}\left(\mathbb{R}^{d}\right)$ so that $\mathcal{P}_{k}=P_{k}$ for $k>0$ and where $\mathcal{P}_{0}$ localizes to frequencies with $|\xi| \leq 2$. For the estimation of $\mathcal{P}_{0} U^{a}$ standard multiplier arguments apply. We also need to consider a similar inhomogeneous Littlewood-Paley decomposition in the $t$ variable, which we denote by $\left\{\mathcal{L}_{j}\right\}_{j=0}^{\infty}$. Then inequality (75) can be rewritten as

$$
\left\|\left(\sum_{j=0}^{\infty} 2^{j \gamma r}\left\|\mathcal{L}_{j}\left[\varpi U^{a} f\right]\right\|_{L^{q}(\mathbb{R})}^{r}\right)^{1 / r}\right\|_{L^{p}\left(\mathbb{R}^{d}\right)} \lesssim\left(\sum_{k} 2^{k(s+a \gamma) p}\left\|\mathcal{P}_{k} f\right\|_{L^{v}\left(\mathbb{R}^{d}\right)}^{p}\right)^{1 / p} .
$$

We claim that there is a constant $M$ for which

$$
\left\|\mathcal{L}_{j}\left[\varpi U^{a} \mathcal{P}_{k} g\right]\right\|_{L^{p}\left(L^{q}\right)} \leq C_{N} \min \left\{2^{-j N}, 2^{-k a N}\right\}\|g\|_{v} \quad \text { whenever }|k a-j| \geq M,
$$

so that for the essential terms $k$ and $j$ are coupled via $|k a-j| \leq M$. This would mean that a $t$ derivative of order $\alpha$ could be traded with an $x$ derivative of order $a \alpha$, so that (79) would follow from (77). Thus it remains to prove (80). Note that for $k>0, j>0$, the convolution kernel of $g \rightarrow \mathcal{L}_{j}\left[\varpi U^{a} \mathcal{P}_{k} g\right](\cdot, t)$ can be written as

$$
\frac{1}{(2 \pi)^{d+1}} \iint\left\{\int \varpi(s) e^{\imath s\left(|\xi|^{a}-\tau\right)} \chi_{1}\left(2^{-j}|\tau|\right) \chi_{1}\left(2^{-k}|\xi|\right) d s\right\} e^{\imath(\langle x, \xi\rangle+t \tau)} d \tau d \xi
$$

and similar formulas hold if either $k=0$ or $j=0$. One checks that if $|k a-j| \gg 1$ then for $\xi$ and $\tau$ in the support of the indicated cutoff functions the inequality $\left.|| \xi\right|^{a}-\tau \mid \geq$ $c \max \left\{|\xi|^{a},|\tau|\right\}$ holds. We perform $N+d+1$ integration by parts in $s$. For $t$ large, we follow this by integrations by parts in $\tau$. This easily yields (80).

The final assertions of the proposition are a consequence of the fact that for $r \leq 1$ the space $B_{1 / q, r}^{q}(\mathbb{R})$ is imbedded in the space of bounded continuous functions. 


\section{REFERENCES}

[1] J. Bourgain, Besicovitch type maximal operators and applications to Fourier analysis, Geom. Funct. Anal., 1 (1991), no. 2, 147-187.

[2] J. Bourgain and L. Guth, Bounds on oscillatory integral operators based on multilinear estimates, preprint, http://arxiv.org/abs/1012.3760.

[3] A. Carbery, The boundedness of the maximal Bochner-Riesz operator on $L^{4}\left(\mathbb{R}^{2}\right)$, Duke Math. J. 50 (1983), no. 2, 409-416.

[4] 287 (1985), no. 2, 673-680.

[5] _ Radial Fourier multipliers and associated maximal functions, Recent progress in Fourier analysis (El Escorial, 1983), 49-56, North-Holland Math. Stud., 111, North-Holland, Amsterdam, 1985.

[6] A. Carbery, G. Gasper and W. Trebels, Radial Fourier multipliers of $L^{p}\left(R^{2}\right)$, Proc. Nat. Acad. Sci. U.S.A. 81 (1984), no. 10, Phys. Sci., 3254-3255.

[7] A. Carbery and A. Seeger, Weighted inequalities for Bochner-Riesz means in the plane, Q. J. Math. 51 (2000), no. 2, 155-167.

[8] M. Christ, On almost everywhere convergence of Bochner-Riesz means in higher dimensions, Proc. Amer. Math. Soc. 95 (1985), 16-20.

[9] A. Córdoba, The Kakeya maximal function and the spherical summation multipliers, Amer. J. Math. 99 (1977), 1-22.

[10] _ A note on Bochner-Riesz operators, Duke Math. J. 46 (1979), no. 3, 505-511.

[11] _ Geometric Fourier analysis, Ann. Inst. Fourier (Grenoble) 32 (1982), no. 3, vii, 215-226.

[12] A. Córdoba and C. Fefferman, A weighted norm inequality for singular integrals, Studia Math. 57 (1976), no. 1, 97-101.

[13] H. Dappa and W. Trebels, On maximal functions generated by Fourier multipliers, Ark. Mat. 23 (1985), no. 2, 241-259.

[14] C. Fefferman, The multiplier problem for the ball, Ann. of Math. (2) 94 (1971), 330-336.

[15] — A note on spherical summation multipliers, Israel J. Math., 15, (1973), 44-52.

[16] C. Fefferman and E.M. Stein, Some maximal inequalities, Amer. J. Math. 93 (1971), 107-115.

[17] $H^{p}$ spaces of several variables, Acta Math. 129 (1972), no. 3-4, 137-193.

[18] J. García-Cuerva and J.L. Rubio de Francia, Weighted norm inequalities and related topics, NorthHolland, 1985.

[19] G. Garrigós and A. Seeger, Characterizations of Hankel multipliers, Math. Ann. 342, no. 1, (2008), 31-68.

[20] Y. Heo, F. Nazarov and A. Seeger, Radial Fourier multipliers in high dimensions, Acta Math. 206 (2011), no.1, 55-92.

[21] S. Igari and S. Kuratsubo, A sufficient condition for $L^{p}$-multipliers, Pacific J. Math. 38 (1971), 85-88.

[22] M. Kaneko and G. Sunouchi, On the Littlewood-Paley and Marcinkiewicz functions in higher dimensions, Tôhoku Math. J. (2) 37 (1985), no. 3, 343-365.

[23] S. Lee, Improved bounds for Bochner-Riesz and maximal Bochner-Riesz operators, Duke Math. J. 122 (2004), 205-232.

[24] Bilinear restriction estimates for surfaces with curvatures of different signs, Trans. Amer. Math. Soc. 358 (2006), no. 8, 3511-3533.

[25] S. Lee, K.M. Rogers and A. Seeger, On space-time estimates for solutions of the Schrödinger equation, in preparation.

[26] A. Miyachi, On some singular Fourier multipliers, J. Fac. Sci. Univ. Tokyo Sect. IA Math., 28 (1981), $267-315$.

[27] G. Mockenhaupt, A. Seeger and C.D. Sogge, Local smoothing of Fourier integral operators and CarlesonSjölin estimates. J. Amer. Math. Soc. 6 (1993), no. 1, 65-130.

[28] M. Pramanik, K.M. Rogers and A. Seeger, A Calderón-Zygmund estimate with applications to generalized Radon transforms and Fourier integral operators, Studia Math. 202 (2011), 1-15.

[29] K.M. Rogers and A. Seeger, Endpoint maximal and smoothing estimates for Schrödinger equations, J. Reine Angew. Math., 640 (2010), 47-66.

[30] J.L. Rubio de Francia, Estimates for some square functions of Littlewood-Paley type, Publ. Sec. Mat. Univ. Autónoma Barcelona 27 (1983), no. 2, 81-108.

[31] _ A Littlewood-Paley inequality for arbitrary intervals, Rev. Mat. Iberoamericana 1 (1985), no. $2,1-14$. 
[32] A. Seeger, On quasiradial Fourier multipliers and their maximal functions, J. Reine Angew. Math. 370 (1986), 61-73.

[33] _ Remarks on singular convolution operators, Studia Math. 97 (1990), 91-114.

[34] __ Endpoint inequalities for Bochner-Riesz multipliers in the plane, Pacific J. Math. 174 (1996), $543-553$.

[35] P. Sjölin, A note on Littlewood-Paley decompositions with arbitrary intervals, J. Approx. Theory 48 (1986), 328-334.

[36] E.M. Stein, Localization and summability of multiple Fourier series, Acta Math. 100 (1958), 93-147.

[37] Maximal functions. I. Spherical means. Proc. Nat. Acad. Sci. U.S.A. 73 (1976), no. 7, 21742175 .

[38] - Some problems in harmonic analysis, in Harmonic analysis in Euclidean spaces (Proc. Sympos. Pure Math., Williams Coll., Williamstown, Mass., 1978), Part 1, 3-20, Amer. Math. Soc., Providence, R.I.

[39] E. M. Stein and G. Weiss, Introduction to Fourier analysis on Euclidean spaces, Princeton Univ. Press, Princeton, NJ, 1971.

[40] G. Sunouchi, On the Littlewood-Paley function $g^{*}$ of multiple Fourier integrals and Hankel multiplier transformations, Tôhoku Math. J. (2) 19 (1967), 496-511.

[41] On the functions of Littlewood-Paley and Marcinkiewicz, Tôhoku Math. J. (2) 36 (1984), no. $4,505-519$.

[42] T. Tao, A sharp bilinear restriction estimate for paraboloids, Geom. Funct. Anal. 13 (2003), 1359-1384.

[43] T. Tao, A. Vargas and L. Vega. A bilinear approach to the restriction and Kakeya conjectures, J. Amer. Math. Soc. 11 (1998), 967-1000.

[44] T. Wolff, An improved bound for Kakeya type maximal functions, Rev. Mat. Iberoamericana 11 (1995), no. $3,651-674$.

Sanghyuk Lee, School of Mathematical Sciences, Seoul National University, Seoul 151742, KOREA

E-mail address: shlee@math.snu.ac.kr

Keith Rogers, Instituto de Ciencias Matematicas CSIC-UAM-UC3M-UCM, Madrid 28049, SPAIN

E-mail address: keith.rogers@icmat.es

Andreas Seeger, Department of Mathematics, University of Wisconsin-Madison, 480 LinCOLn Drive, Madison, WI, 53706, USA

E-mail address: seeger@math.wisc.edu 